\title{
Parallel selection on ecologically relevant gene functions in the transcriptomes of highly diversifying salmonids
}

\author{
Kevin Schneider ${ }^{1}$, Colin E. Adams ${ }^{1,2}$ and Kathryn R. Elmer ${ }^{1 *}$ (D)
}

\begin{abstract}
Background: Salmonid fishes are characterised by a very high level of variation in trophic, ecological, physiological, and life history adaptations. Some salmonid taxa show exceptional potential for fast, within-lake diversification into morphologically and ecologically distinct variants, often in parallel; these are the lake-resident charr and whitefish (several species in the genera Salvelinus and Coregonus). To identify selection on genes and gene categories associated with such predictable diversifications, we analysed 2702 orthogroups (4.82 Mbp total; average 4.77 genes/orthogroup; average 1783 bp/orthogroup). We did so in two charr and two whitefish species and compared to five other salmonid lineages, which do not evolve in such ecologically predictable ways, and one non-salmonid outgroup.

Results: All selection analyses are based on Coregonus and Salvelinus compared to non-diversifying taxa. We found more orthogroups were affected by relaxed selection than intensified selection. Of those, 122 were under significant relaxed selection, with trends of an overrepresentation of serine family amino acid metabolism and transcriptional regulation, and significant enrichment of behaviour-associated gene functions. Seventy-eight orthogroups were under significant intensified selection and were enriched for signalling process and transcriptional regulation gene ontology terms and actin filament and lipid metabolism gene sets. Ninety-two orthogroups were under diversifying/positive selection. These were enriched for signal transduction, transmembrane transport, and pyruvate metabolism gene ontology terms and often contained genes involved in transcriptional regulation and development. Several orthogroups showed signs of multiple types of selection. For example, orthogroups under relaxed and diversifying selection contained genes such as ap $1 \mathrm{~m} 2$, involved in immunity and development, and s/c6a8, playing an important role in muscle and brain creatine uptake. Orthogroups under intensified and diversifying selection were also found, such as genes syn3, with a role in neural processes, and ctsk, involved in bone remodelling.

Conclusions: Our approach pinpointed relevant genomic targets by distinguishing among different kinds of selection. We found that relaxed, intensified, and diversifying selection affect orthogroups and gene functions of ecological relevance in salmonids. Because they were found consistently and robustly across charr and whitefish and not other salmonid lineages, we propose these genes have a potential role in the replicated ecological diversifications.
\end{abstract}

Keywords: Molecular evolution, Adaptation, Freshwater fishes, Diversification, Relaxed selection, Selective pressure, Purifying selection, Positive selection, Transcriptomics, Gene ontology

\footnotetext{
* Correspondence: Kathryn.Elmer@glasgow.ac.uk

${ }^{1}$ Institute of Biodiversity, Animal Health \& Comparative Medicine, College of

Medical, Veterinary \& Life Sciences, University of Glasgow, Glasgow G12 8QQ,

UK

Full list of author information is available at the end of the article
}

(c) The Author(s). 2019 Open Access This article is distributed under the terms of the Creative Commons Attribution 4.0 International License (http://creativecommons.org/licenses/by/4.0/), which permits unrestricted use, distribution, and reproduction in any medium, provided you give appropriate credit to the original author(s) and the source, provide a link to the Creative Commons license, and indicate if changes were made. The Creative Commons Public Domain Dedication waiver (http://creativecommons.org/publicdomain/zero/1.0/) applies to the data made available in this article, unless otherwise stated. 


\section{Background}

Identifying the molecular mechanisms underlying adaptive phenotypic divergence is a central challenge for evolutionary biology; a key first step is to detect genes under selection rather than reflecting background neutral evolutionary processes. Parallel or convergent evolution at the molecular level may, or may not, be associated with phenotypic parallelisms across species, but the idea remains compelling [1-3] and has been an important analytical framework to advance research in non-model systems [4-6]. Molecular parallelism or convergence can be inferred either from nucleotide sitespecific changes [5, 7-9] or at a higher level, in the sense of similar genes being targeted by similar selective forces [10-13].

Fishes have proven a powerful ecological and evolutionary group for comparisons of genes under selection and that are associated with ecological and evolutionary novelty. Sticklebacks, for example, have become a model group of repeated ecological adaptation of Holarctic marine and freshwater distribution [14-16]. In cichlid fishes, adaptive potential and highly malleable phenotypes are spread throughout the family. In some cases, it has been shown that relaxed selection [17-20] or positive selection $[21,22]$ correlate with phenotypic diversification. However, ecological opportunity differs dramatically among cichlid lineages [23-27], which makes it difficult to pinpoint taxa in which adaptive potential is elevated due to a shared genetic toolset $[3,6]$. In contrast, freshwater lake-resident salmonids of different species and genera have similar ecological opportunity and commonly sympatric distributions across the northern hemisphere [28-30]. Furthermore, the freshwater habitats of northern fishes were all colonised on a similar postglacial timescale [30,31], unlike the dramatically different and complex colonisation histories of cichlids [4, 32-34].

Salmonid fishes are increasingly used as model organisms in evolutionary research, because of their ecological diversity, economic value, and replicated evolution of distinct ecomorphs and traits in some taxa, such as depth specialisation and alternative migratory tactics [35-42]. Two salmonid genera in particular, the whitefishes (Coregonus) and the charrs (Salvelinus), are not sister taxa but exhibit parallel (or convergent) adaptive tendencies in freshwaters across the northern hemisphere. They have repeatedly diverged into various within-lake ecomorphs along the depth axis over short evolutionary time spans that are unmatched in any other salmonid species [2, 43-47]. The evolutionary and molecular basis for why Coregonus and Salvelinus show such a high degree of ecomorphological adaptability while other salmonid species do not is, however, unknown [39, 46, 47].
Determining how single and combined effects of selection act at the molecular level is facilitated by new analytical tools [48-50]. These selective processes are associated with adaptive evolution in different ways and are most powerful when linked with known lineagespecific phenotype changes or phenotypic diversification $[51,52]$. Two selective processes - relaxed and intensified selection - are on opposite ends of the spectrum. Relaxed selection decreases the selective constraints of a gene and can lead to the accumulation of nonsynonymous substitutions and consequently changes in the amino acid sequence of a protein. By releasing a gene of selective constraints, relaxed selection can potentially foster phenotypic novelty, plasticity, and evolutionary innovation $[4,19,49,53]$. In contrast, intensified selection increases selective constraints but can also manifest as positive intensified selection leading to more differences at some sites of a gene $[54,55]$. Intensified selection implies changes to a gene have strong fitness consequences [49]. Additionally, lineage-specific episodic diversifying selection, or positive selection, will leave other signatures at the sequence level, such as more nonsynonymous changes than expected under neutrality at a subset of positions in a gene on some branches in the phylogenetic tree (i.e., branch-site model) [50, 56]. While relaxed and intensified selection are antithetical, in either case diversifying (positive) selection can simultaneously act at a proportion of sites in a gene [57, 58].

It has long been proposed that the propensity for ecological speciation in some salmonid lineages is associated with shared patterns of relaxed or diversifying selection on ecologically relevant genes and gene function terms $[2,3,19,39,59,60]$. We focus on the well-characterised and richly diversifying genera Coregonus and Salvelinus, which show repeated within-lake divergences into distinct ecomorphs across the northern hemisphere $[46,47]$. The Coregonus species assessed are lake resident at least since postglacial times and have high rates of within-lake adaptive divergence $[45,46,61]$. Salvelinus species are mostly freshwater residents and have undergone frequent adaptive divergence into ecomorphs along the depth axis, predominantly within lakes [38, 47, 62, 63]. Representatives of all other major salmonid lineages, Oncorhynchus, Salmo, and Thymallus, were also included in the dataset; these are generally riverine or anadromous genera that do not extensively diversify within lakes $[64,65]$. By assessing consistency across two non-sister lineages, Coregonus and Salvelinus, our approach mitigates against false positives. The focus on orthogroups within and across species, rather than single genes, alleviates the problem of differing relaxation of selective constraint in duplicated compared to non-duplicated or rediploidised genes [66], which is particularly important in salmonids due to the whole-genome duplication 
(WGD) that their common ancestor experienced 80103 Mya [67-69].

Here, we use a genome-wide comparative approach to test for shared evidence of selection at particular categories of genes, gene functions, and gene ontology terms in the two highly diversifying lineages, Coregonus and Salvelinus, relative to all other major salmonid lineages. We test a comprehensive suite of 2702 orthologous protein-coding gene sets (orthogroups) for signals of parallel relaxed, intensified, and diversifying/positive selection in Coregonus and Salvelinus (average of 4.77 genes per orthogroup; $4.82 \mathrm{Mbp}$ in total; average of 1783 aligned bp per orthogroup). By distinguishing among different kinds of selection in replicate across two independent lineages, our approach can pinpoint the action of selective pressure more accurately. We find that different types of selection target different gene sets and functions in salmonids, with novel and established ecological relevance for repeated, parallel diversification potential.

\section{Results}

Selection parameter distribution and number of orthogroups under selection

Shared molecular response to selection in two whitefish and two charr species was inferred relative to six background species (five salmonids and one pike, Fig. 1). The selection parameter $k$ in whitefish and charr, ranging from 0 (very relaxed) to 50 (very intensified), had a median value of 0.992 across orthogroups and was significantly different from the neutral expectation of 1 (Wilcoxon signed-rank test: $V=1,995,900, p=8.859 \mathrm{E}$ 06). Visually, there was an excess of orthogroups with $k$ close to 0 , indicating a high number of orthogroups under pronounced relaxed selection (Fig. 2). The number of orthogroups with $k<1$ (1387; relaxed selection prevailing) was slightly higher than the number of orthogroups with $k>1$ (1308; intensified selection prevailing), but not significantly so (Fisher's Exact Test, $p=0.288$ ).

On 2702 orthogroups in the final dataset we conducted analyses of relaxed and intensified selection (in RELAX) and diversifying/positive selection (in aBSREL with branch-site model). We inferred 138 orthogroups to be under relaxed selection $(k<1$, false discovery rate $($ FDR $)<0.10)$ in either Coregonus or Salvelinus, of which 122 were found in both Coregonus and Salvelinus. On the other hand, 105 orthogroups showed signals of intensified selection $(k>1, \mathrm{FDR}<0.10)$, of which 78 included both Coregonus and Salvelinus. The number of relaxed orthogroups in Coregonus and Salvelinus was significantly higher compared to the number of intensified orthogroups (one-sided Fisher's Exact Test, $p=$ 0.035). Of the 2702 orthogroups, branch-site selection analyses inferred 111 orthogroups as being under significant diversifying/positive selection $(F D R<0.10)$, of which 92 included both Coregonus and Salvelinus. Thus, these orthogroups harbour a proportion of sites with significantly elevated $\mathrm{dN} / \mathrm{dS}(=\omega)$ values in at least one of

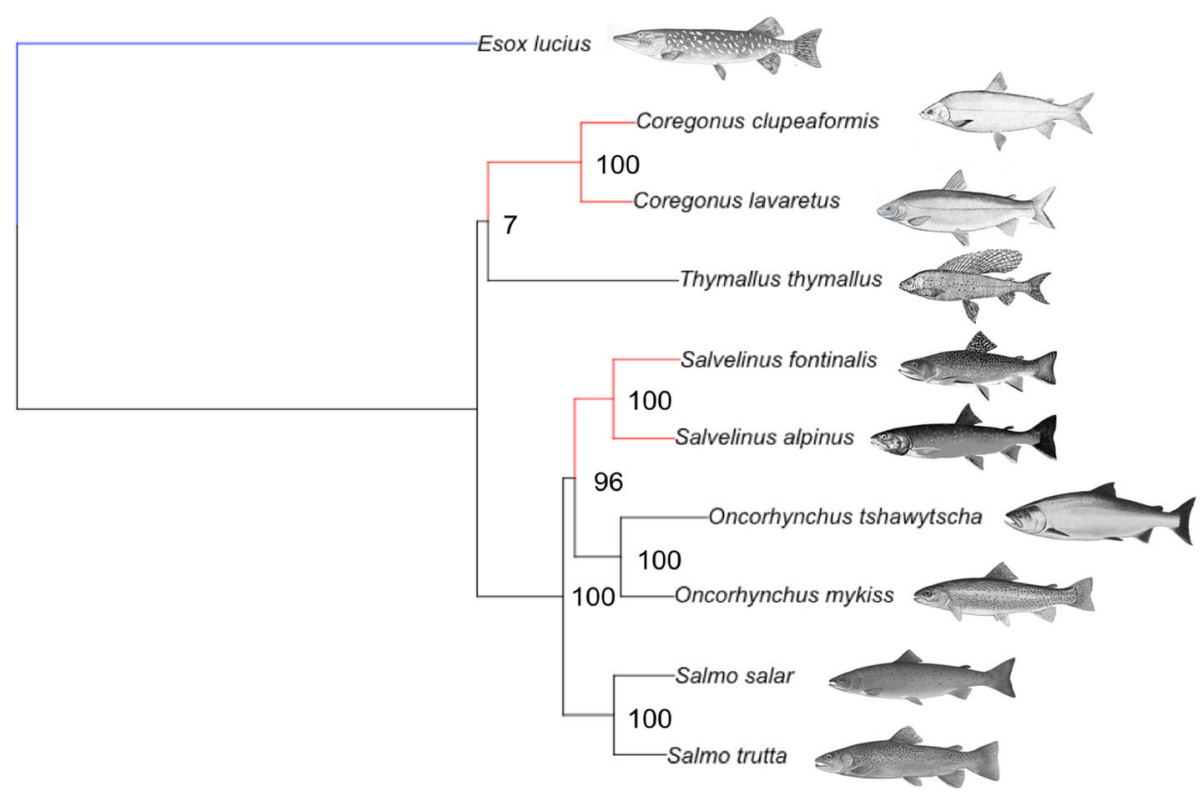

Fig. 1 Maximum-likelihood phylogenetic tree of nine salmonid species (foreground taxa Coregonus and Salvelinus in red) and outgroup northern pike (Esox lucius; in blue). Node support values are bootstrap values from 1000 bootstrap replications. Branch lengths correspond to the number of substitutions per site. All pictures used here are under public domain. Coregonus and Salvelinus are two genera with exceptional ability for repeated, rapid diversification into ecomorphs within lakes that is unmatched in other salmonid taxa [2, 43-47] 


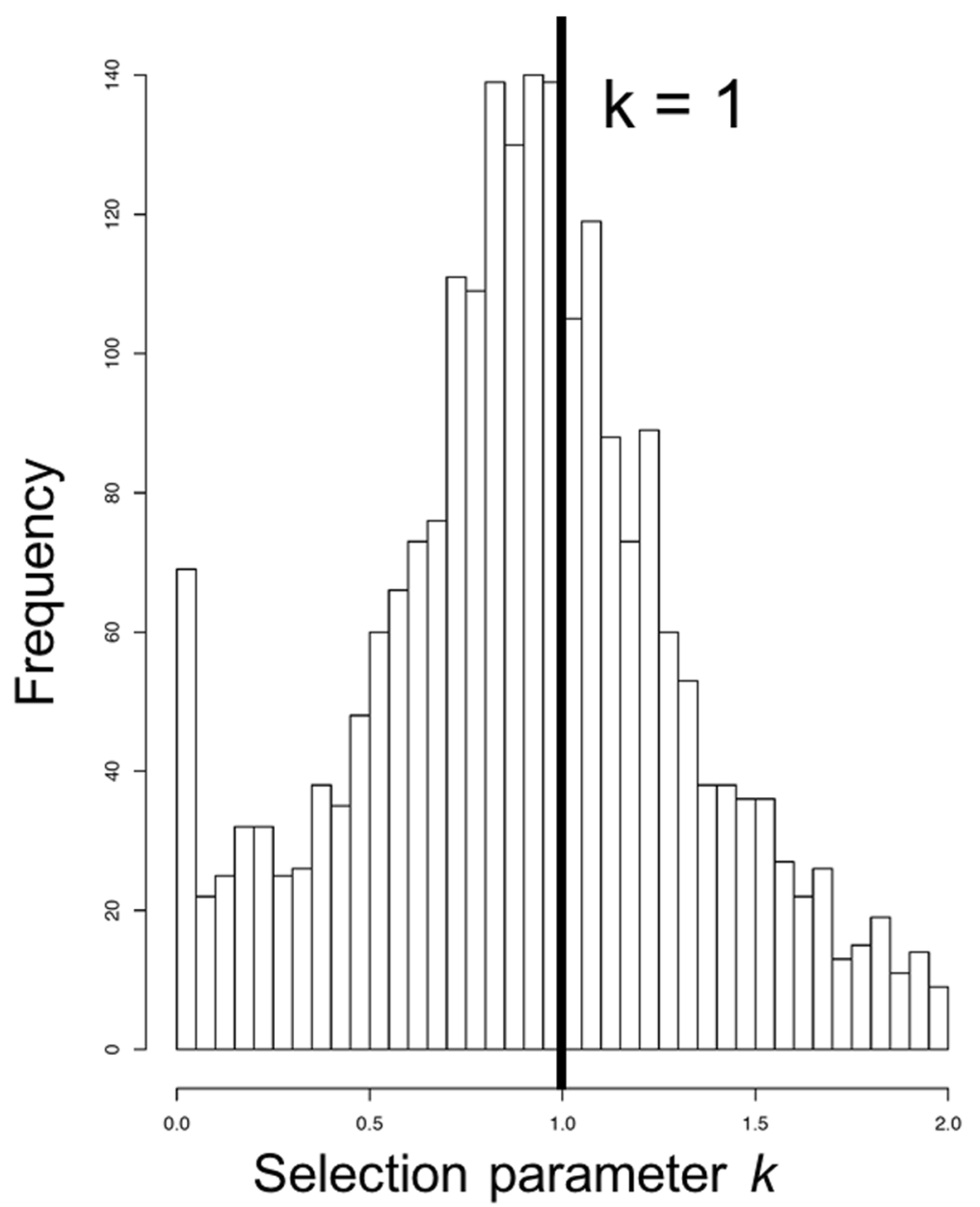

Fig. 2 Histogram of the distribution of selection parameter $k$ values (exponent of dN/dS ratio, i.e., $k$ in $\omega^{k}$ ) from RELAX analysis in the 2702 orthogroups. The values shown are for Coregonus and Salvelinus compared to the other five salmonid species and the outgroup (pike). The neutral expectation of $k=1$ is shown as a vertical line. $k$ values above 2 are omitted for visibility purposes in the above plot (416 orthogroups have $k$ values above 2 ; the maximum possible $k$ value is 50 ; see Additional file 1 for total set of $k$ values)

the foreground branches leading to Coregonus or Salvelinus taxa.

After averaging selection parameter values for each gene ontology (GO) term, 13 of $1478 \mathrm{GO}$ terms showed significant deviations from the null expectation of $k=1$ (Wilcoxon signed-rank tests: $p<0.05$; Fig. 3). Eight of these had significantly elevated $k$ values, indicating intensified selection. The other five had significantly lowered $k$ values, which is evidence for relaxed selection. The GO term enrichment results agreed with the general shift of selection (distribution of $k$ ) in all orthogroups. The GO terms 'carbohydrate metabolic process' and 'obsolete acylcarrier-protein biosynthetic process', for example, were also present in the orthogroups under intensified selection. The 'ATPase activity' and 'proton transmembrane transport' GO terms were also found among orthogroups under relaxed selection. Other deviating GO terms were 'DNA repair' and 'protein deubiquitination', with evidence for intensified selection, and 'exocytosis' and 'protein dephosphorylation', with evidence for relaxed selection (Fig. 3).

\section{Gene functions under relaxed selection}

Blast2GO annotation and UniProt/Swiss-Prot literature research on the orthogroups under relaxed selection identified gene functions with potential relevance for the diversification process in charr and whitefish. Such functions included visual perception (e.g., 'peripherin-2-like'), gene and gene product regulation (e.g., 'E3 ubiquitin-protein ligase RNF128-like'), lipid metabolism (e.g., 'calcium-independent phospholipase A2-gamma-like'), muscle and heart growth (e.g., 'dual specificity protein phosphatase 6'), locomotion (e.g., 'serine/threonine-protein phosphatase PP1-beta catalytic subunit'), and immunity (e.g., 'adaptorrelated protein complex 1', 'natterin-3-like'), but also genes with a role for various nervous system processes 


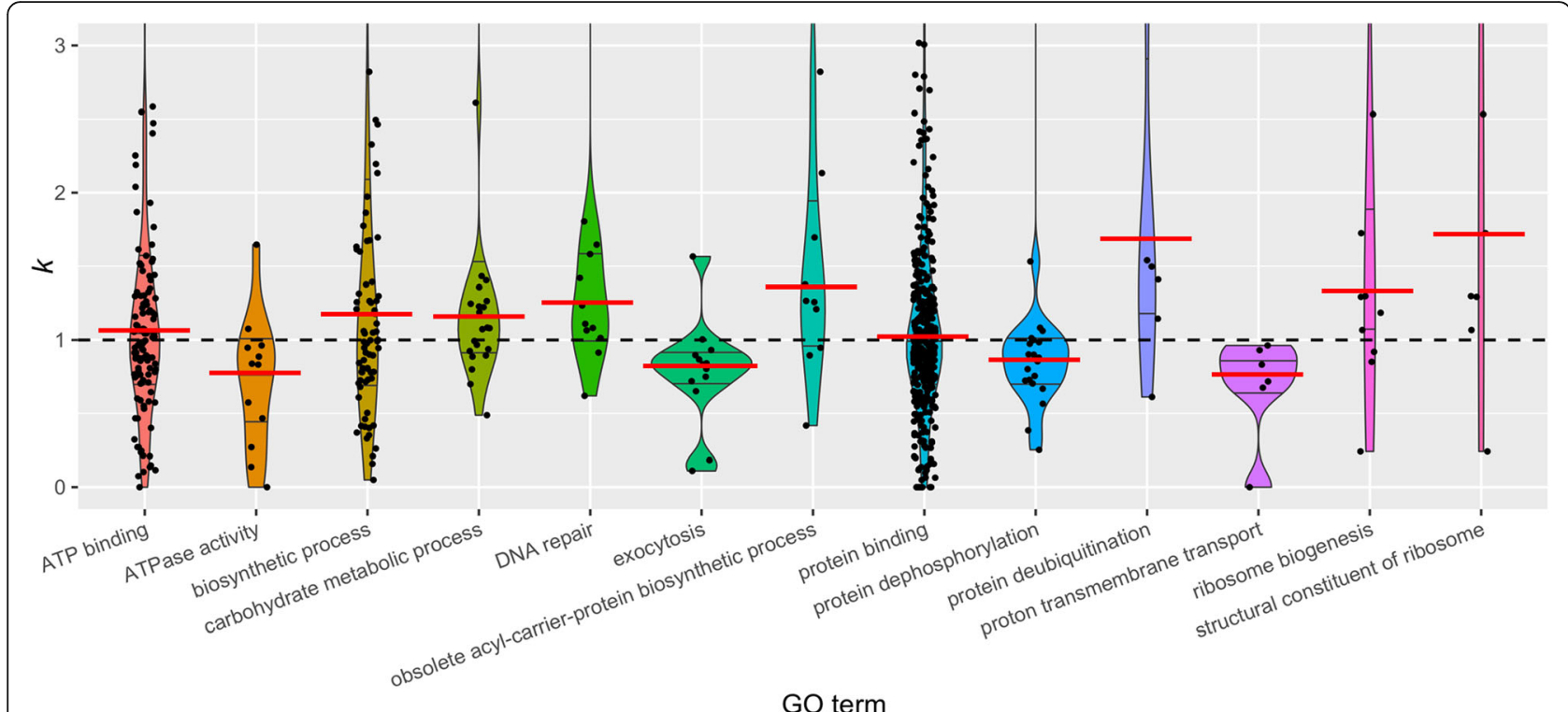

Fig. 3 Selection parameter $k$ distributions for the gene ontology (GO) terms that deviated from the null expectation in the total set of 2702 orthogroups. $k$ values are selection parameter values (exponent of $\mathrm{dN} / \mathrm{dS}$ ratio, i.e., $k$ in $\omega^{k}$ ) in Coregonus and Salvelinus compared to the five other salmonid species and the outgroup (pike). The red horizontal bars indicate the medians of $k$

(e.g., 'POU domain, class 4, transcription factor 3-like'; results of relaxed and intensified selection analyses: Additional file 1).

We observed compelling trends of GO term enrichment (one-tailed Fisher's Exact Tests, uncorrected $p<$ 0.05 but FDR $>0.10$ ) in the orthogroups under relaxed, intensified, and diversifying selection that largely agree with the research literature on the genes contained in those orthogroups (Fig. 4, Table 1). We found the 122 orthogroups under significant relaxed selection in Coregonus and Salvelinus were enriched for a total of $11 \mathrm{GO}$ terms associated with transcriptional regulation, serine family amino acid metabolism, lipid metabolism, and oxidoreductase activity, amongst others (Table 1). The REVIGO redundancy analysis results showed transcriptional regulation, serine family amino acid metabolism, lipid metabolism, and acrosome reaction to be among the few non-redundant GO terms (frequency and significance plot of non-redundant GO terms: Fig. 4a, includes clustering by semantic similarity). Transcriptional regulation and serine family amino acid metabolism were the most frequent non-redundant GO terms. In total, six of $11 \mathrm{GO}$ terms were found to be non-redundant.

Among the top ten enriched functions in relaxed orthogroups in both Coregonus and Salvelinus, behaviour and many neural function GO terms and KEGG pathways were found in gene set enrichment analyses (Fig. 5). This is in agreement with the neural process orthogroups and serine family amino acid metabolism GO terms obtained in the GO term enrichment analysis above. The behaviour gene set was the only gene set that was significantly enriched after FDR correction (Fig. 5). Other overrepresented functions included, for example, negative regulation of signalling, urogenital system development, the peroxisome pathway, vascular smooth muscle contraction, and the AGE-RAGE signalling pathway, which plays a major role in inflammation and infection.

\section{Gene functions under intensified selection}

The gene functions of the 78 orthogroups under intensified selection in both Coregonus and Salvelinus (results of relaxed and intensified selection analyses: Additional file 1) were found from literature search to be frequently involved in functions relevant for lipid and carbohydrate metabolism (e.g., 'acetyl-CoA carboxylase beta' and 'endoplasmic reticulum mannosyl-oligosaccharide 1,2-alphamannosidase-like', respectively) as well as neurological and bone development (e.g., neurological development: 'synapsin-3', bone development: 'cathepsin K precursor' and 'paired like homeodomain 1').

The orthogroups under intensified selection in Coregonus and Salvelinus were enriched for transcriptional regulation GO terms, but also for those associated with ubiquitine-related processes and steroid hormone receptor activity, amongst others. A total of $18 \mathrm{GO}$ terms were overrepresented (Table 1). Transcriptional regulation and several signalling processes were the only highfrequency GO terms among the few non-redundant GO terms in the REVIGO analysis (frequency and significance plot of non-redundant GO terms: Fig. 4b, includes clustering by semantic similarity). In total, nine of 18 

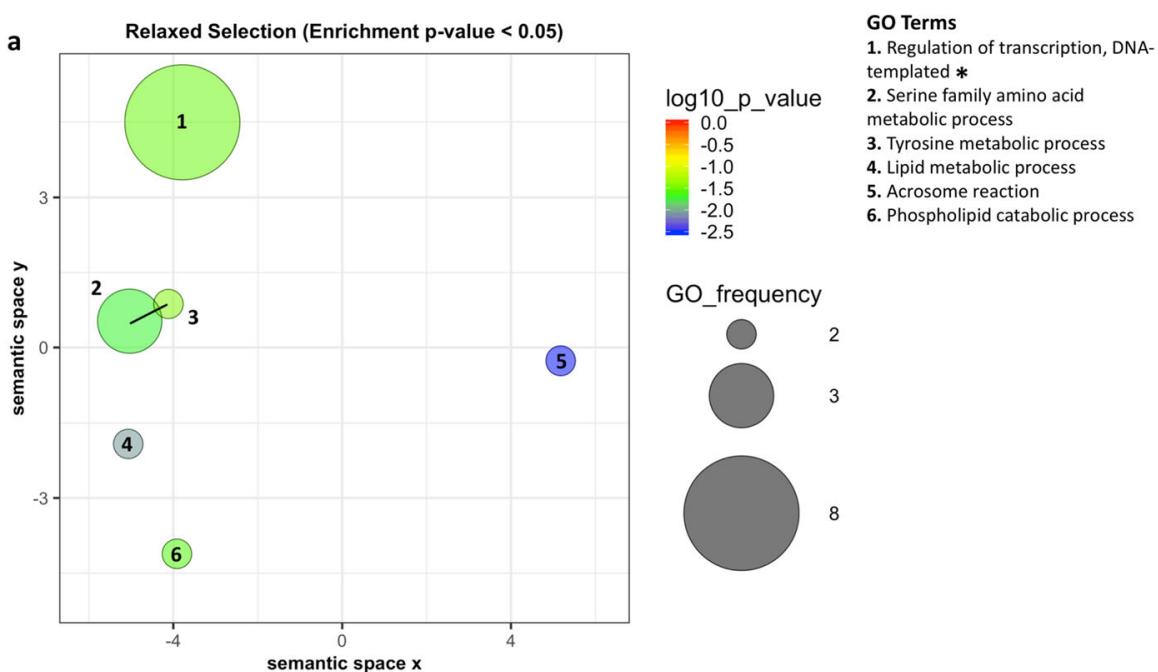

GO Terms

1. Regulation of transcription, DNA-

templated *

3. Tyrosine metabolic process

4. Lipid metabolic process

6. Phospholipid catabolic process

\section{GO_frequency}

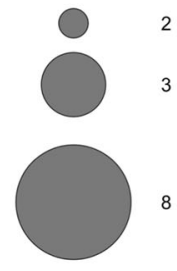

b Intensified Selection (Enrichment p-value $<0.05$ )

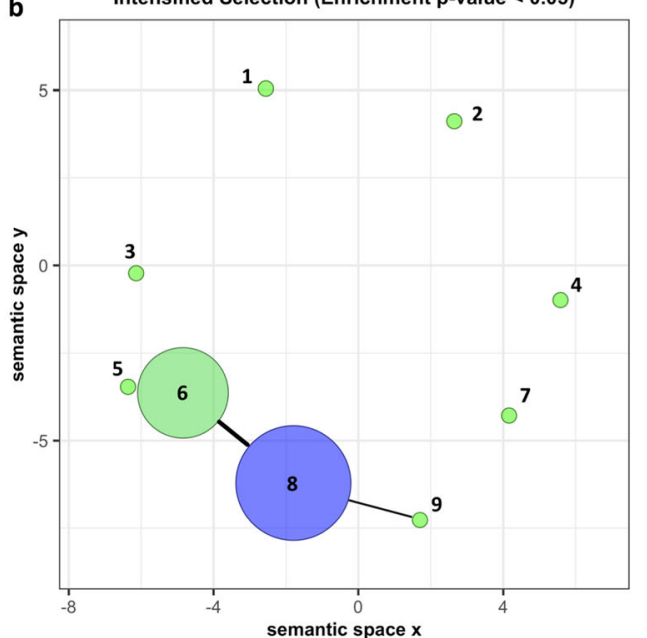

GO Terms

1. Adrenal gland development

$\begin{array}{ll} & \\ \log 10 \_p \_v a l u e & \text { 2. Actin filament severing } \\ & \text { 3. Regulation of NIK/NF-kappaB }\end{array}$

$\begin{array}{ll}0.0 & \text { 3. Regulation of NIK/NF-ka } \\ -0.5 & \text { s. Ribosomal large subunit } \\ -1.0 & \text { biogenesis }\end{array}$

5. Apoptotic signaling pathway

6. Signal transduction

7. Histone $\mathrm{H} 3-\mathrm{K} 4$ demethylation

$-2.0$

8. Regulation of

GO_frequency templated $*$

$\begin{array}{lll}0 & 1 & \text { 9. Retinoic acid biosynthesis }\end{array}$

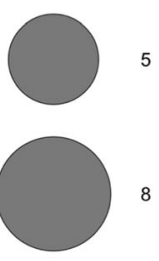

Diversifying Selection (Enrichment $p$-value $<0.05$ )

1. Transmembrane transport

2. Urea transmembrane transpor

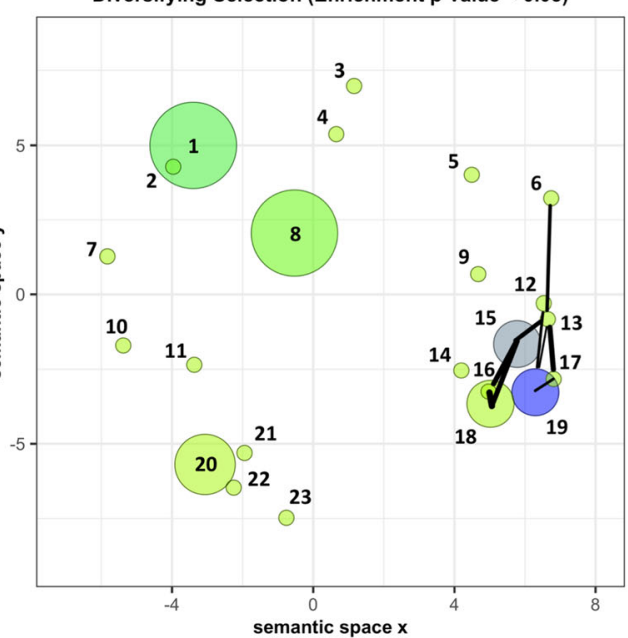

$\begin{array}{ll}\log 10 \_p \_v a l u e & \begin{array}{l}\text { 3. Proteasome regulatory particle } \\ \text { assembly } \\ 0\end{array} \\ \text { 4. Histone H3 deacetylation }\end{array}$

5. RNA splicing
-1 6.5-methylcytosine catabolic process

$-2 \quad$ 7. Sulfate assimilation

8. Proteolysis

9. DNA demethylation

10. Antigen processing and

GO_frequency presentation of endogenous peptide

antigen via MHC class I
11. Complement activation

$\begin{array}{ll}1 & \text { 11. Complement activation } \\ 2 & \text { 12. Proline biosynthesis }\end{array}$

13. 10 -formyltetrahydrofolate catabolic process

process
14. Glycogen metabolic process

14. Glycogen metabolic process
15. Lipid catabolic process

15. Lipid catabolic process

16. Phospholipid metabolic process

17. Malate metabolic process

18. Glycerolipid metabolic proc

20. Intracellular signal transduction

21. Regulation of Wnt signaling

pathway

22. Transforming growth factor beta

receptor signaling pathway

23. Calcium-mediated signaling

Fig. 4 (See legend on next page.) 
(See figure on previous page.)

Fig. 4 Gene Ontology $(G O)$ terms with trends of overrepresentation $(p<0.05)$ in $\mathbf{a}$ the orthogroups under relaxed selection (FDR $<0.10)$, b the orthogroups under intensified selection (FDR $<0.10$ ), and $\mathbf{c}$ the orthogroups under diversifying selection (FDR $<0.10)$. The orthogroups used are present in both Coregonus and Salvelinus. Bubble colour in the indicated colour spectrum corresponds to the $\log _{10} p$-value of overrepresentation (blue = higher significance, red = lower significance). Bubble size corresponds to the frequency of a GO term in the orthogroups under selection. Highly similar GO terms are linked by edges in the graph, where the line width indicates the degree of similarity. The bubble placement corresponds to the position along two semantic space axes based on SimRel clustering as described in Material \& methods [70]. Asterisks indicate overlap with enriched GO terms in orthogroups under other types of selection

GO terms remained after the REVIGO redundancy analysis.

In the gene set enrichment analysis of all intensified selection orthogroups present in Coregonus and Salvelinus, the 'actin filament-based process' GO term, the 'spliceosome' and several signalling KEGG pathways were among the top enriched functions (Fig. 5). Other functions included 'cellular protein-containing complex assembly', 'fatty acid elongation', 'progesterone-mediated oocyte maturation', and 'steroid biosynthesis'. Overall, the gene enrichment results (Fig. 5) mostly agree with the GO term overrepresentations (Fig. 4b).

\section{Gene functions under diversifying selection}

A large number of the 92 orthogroups under diversifying selection in Coregonus and Salvelinus were found in literature search to contain genes involved in regulation of gene expression, signal transduction and transmembrane transporter genes, but also immunity-related genes and a gene of the FOX set of genes, 'FOX I1-ema', a tissue-specific splicing factor important in otic placode formation and jaw development in zebrafish [71] (orthogroups under diversifying selection: Additional file 2, includes associated GO terms).

Orthogroups under diversifying selection were enriched for GO terms associated with transmembrane transport, phospholipid metabolic processes, acetyl-CoA carboxylase activity, various lipid metabolic processes, regulation of Wnt signalling pathway, and RNA splicing, amongst others (Fig. 4c, Table 1). A total of $47 \mathrm{GO}$ terms were overrepresented, of which 23 remained after the REVIGO redundancy analysis. Pyruvate metabolism, several signal transduction processes, lipid metabolism, and transmembrane transport processes were shown to be amongst the non-redundant GO terms in the REVIGO analysis (frequency and significance plot of non-redundant GO terms: Fig. 4c, includes clustering by semantic similarity). Compared to the orthogroups under relaxed or intensified selection (Fig. 4a,b), the orthogroups under diversifying selection included a higher number of rather dissimilar low-frequency GO terms, apart from a cluster of similar metabolic GO terms (Fig. 4c). Only one GO term, 'DNA binding', was underrepresented $(p<0.05)$; with zero occurrences in the orthogroups under diversifying selection in Coregonus and Salvelinus but 77 occurrences in all other orthogroups.

\section{Overlap between selection types}

We identified nine orthogroups that showed both signals of relaxed selection (RELAX) and diversifying selection (aBSREL) and 12 orthogroups that showed both signals of intensified selection (RELAX) and diversifying selection (aBSREL) (Fig. 6a, Table 2). The overlap between orthogroups under relaxed and diversifying selection was higher than expected by chance, but not significantly so (hypergeometric expectation: 3.6 vs observed 9; onetailed Fisher's Exact Test: $p=0.126$ ). The overlap between orthogroups under intensified and diversifying selection was significantly higher than expected by chance (hypergeometric expectation: 2.1 vs observed 12; onetailed Fisher's Exact Test: $p=0.004$ ).

Based on UniProt/Swiss-Prot gene information, the orthogroups with both signals of relaxed and diversifying selection are associated with functions such as immunity (5 of 9 orthogroups, e.g., 'adaptor-related protein complex 1 , mu 2 subunit (ap1m2)'), the nervous system (4 of 9 orthogroups, e.g., 'protein kinase C epsilon type-like (prkce)'), muscle function (2 of 9 orthogroups, e.g., 'solute carrier family 6 (neurotransmitter transporter), member 8 $($ slc6a8)'), blood pressure (1 of 9 orthogroups, 'endoplasmic reticulum aminopeptidase 1-like (LOC106570844)'), and transcriptional regulation (1 of 9 orthogroups, 'probable histone deacetylase 1-B (hdac1-b)') (Table 2). This is in agreement with the more general GO term functions inferred using Blast2GO and associated tools (Fig. 6b biological process GO terms, Table 2), such as neurotransmitter transport, calcium-mediated signalling, antigen presentation, regulation of blood pressure, and serine family amino acid metabolism.

Based on UniProt/Swiss-Prot gene information, orthogroups with both signals of intensified and diversifying selection are associated with transcriptional regulation (4 of 12 orthogroups, e.g., 'paired amphipathic helix protein Sin3a-like $\left.(\sin 3 a)^{\prime}\right)$, lipid metabolism $(3$ of 12 orthogroups, e.g., 'acetyl-CoA carboxylase beta (acacb)'), nervous system function (3 of 12 orthogroups, e.g., 'synapsin-3 (syn3)'), carbohydrate metabolism (2 of 12 orthogroups, e.g., 'alpha-2,8-sialyltransferase 8F-like 
Table 1 Over- and underrepresented terms from a GO enrichment analysis. Cor = Coregonus, Salv = Salvelinus, OGs = orthogroups

\begin{tabular}{llllll}
\hline GO category & $p$-value & $\begin{array}{l}p \text {-value } \\
\text { (Cor + Salv) }\end{array}$ & $\begin{array}{l}\text { \# in OGs under } \\
\text { selection }\end{array}$ & $\begin{array}{l}\text { \# in reference } \\
\text { OGs }\end{array}$ & $\begin{array}{l}\text { \# in OGs } \\
\text { under selection } \\
\text { (Cor + Salv) }\end{array}$
\end{tabular}

Overrepresented GO terms - relaxed selection

PTW/PP1 phosphatase complex

acrosome reaction

integral component of membrane

transcription factor complex

regulation of transcription, DNA-templated

DNA-binding transcription factor activity

regulation of transcription by RNA polymerase II

lipid metabolic process

protein serine/threonine kinase activity

protein tyrosine phosphatase activity

phospholipid catabolic process

tyrosine metabolic process

oxidoreductase activity, acting on paired donors, with incorporation or reduction of molecular oxygen

serine family amino acid metabolic process

nucleus

Overrepresented GO terms - intensified selection

protein deubiquitination

regulation of transcription, DNA-templated

thiol-dependent ubiquitinyl hydrolase activity

DNA binding

steroid hormone receptor activity

acetyl-CoA carboxylase activity

acetyl-CoA carboxylase complex

Lys48-specific deubiquitinase activity

Sin3 complex

histone H3-K4 demethylation

retinoic acid biosynthetic process

NADP-retinol dehydrogenase activity

catalase activity

obsolete peroxidase reaction

response to oxidative stress

methane metabolic process

hydrogen peroxide catabolic process

palmitoyltransferase activity

beta1-adrenergic receptor activity

adenylate cyclase-activating $\mathrm{G}$ protein-coupled receptor signaling pathway

positive regulation of heart contraction

adrenergic receptor signaling pathway

adrenal gland development

corticotropin-releasing hormone binding
2.55E-03 3.03E-03 2

2.55E-03 3.03E-03 2

$3.96 \mathrm{E}-03 \quad 1.77 \mathrm{E}-02 \quad 21$

4.59E-03 5.52E-02 11

6.29E-03 3.12E-02 10

$1.22 \mathrm{E}-02 \quad 5.98 \mathrm{E}-02 \quad 9$

$1.35 \mathrm{E}-02 \quad 2.20 \mathrm{E}-01 \quad 4$

1.43E-02 8.76E-03 2

2.00E-02 1.48E-02 3

2.30E-02 2.71E-02 2

2.30E-02 2.71E-02 2

3.34E-02 3.92E-02 2

3.34E-02 2.71E-02 2

3.70E-02 1.96E-02 3

4.42E-02 6.75E-02 10

1.73E-03 1.33E-01 3

$2.17 \mathrm{E}-03 \quad 5.18 \mathrm{E}-03 \quad 10$

8.36E-03 1.02E-01 2

$2.48 \mathrm{E}-02 \quad 1.68 \mathrm{E}-02 \quad 8$

2.71E-02 2.29E-02 2

3.85E-02 3.52E-02 1

3.85E-02 3.52E-02 1

3.85E-02 1.00E+00 1

3.85E-02 3.52E-02 1

3.85E-02 3.52E-02 1

3.85E-02 3.52E-02 1

3.85E-02 3.52E-02 1

3.85E-02 1.00E+00 1

3.85E-02 1.00E+00 1

3.85E-02 1.00E+00 1

3.85E-02 1.00E+00 1

3.85E-02 1.00E+00 1

3.85E-02 3.52E-02 1

3.85E-02 1.00E+00 1

3.85E-02 1.00E+00 1

3.85E-02 1.00E+00 1

3.85E-02 1.00E+00 1

3.85E-02 3.52E-02 1

3.85E-02 1.00E $+00 \quad 1$ (Cor + Salv)

\section{0}

68

60

57

14

1

7

3

3

4

3

8

84

3

70

2

70

5

0

0

0

0

0

0

0

0

0

0

0

0

0

0

0

0

0

0

0 
Table 1 Over- and underrepresented terms from a GO enrichment analysis. Cor = Coregonus, Salv = Salvelinus, OGs = orthogroups (Continued)

\begin{tabular}{|c|c|c|c|c|c|c|}
\hline GO category & $p$-value & $\begin{array}{l}p \text {-value } \\
(\text { Cor + Salv) }\end{array}$ & $\begin{array}{l}\text { \# in OGs under } \\
\text { selection }\end{array}$ & $\begin{array}{l}\text { \# in reference } \\
\text { OGs }\end{array}$ & $\begin{array}{l}\text { \# in OGs } \\
\text { under selection } \\
\text { (Cor + Salv) }\end{array}$ & $\begin{array}{l}\text { \# in reference } \\
\text { OGs (Cor + Salv) }\end{array}$ \\
\hline apoptotic signaling pathway & $3.85 \mathrm{E}-02$ & $3.52 \mathrm{E}-02$ & 1 & 0 & 1 & 0 \\
\hline regulation of NIK/NF-kappaB signaling & 3.85E-02 & $3.52 \mathrm{E}-02$ & 1 & 0 & 1 & 0 \\
\hline iron-sulfur cluster binding & $3.85 \mathrm{E}-02$ & $3.52 \mathrm{E}-02$ & 1 & 0 & 1 & 0 \\
\hline actin filament severing & 3.85E-02 & $3.52 \mathrm{E}-02$ & 1 & 0 & 1 & 0 \\
\hline dystrophin-associated glycoprotein complex & 3.85E-02 & $3.52 \mathrm{E}-02$ & 1 & 0 & 1 & 0 \\
\hline hexosaminidase activity & $3.85 \mathrm{E}-02$ & $1.00 \mathrm{E}+00$ & 1 & 0 & 0 & 0 \\
\hline hyaluronic acid binding & 3.85E-02 & $3.52 \mathrm{E}-02$ & 1 & 0 & 1 & 0 \\
\hline ribosomal large subunit biogenesis & $3.85 \mathrm{E}-02$ & $3.52 \mathrm{E}-02$ & 1 & 0 & 1 & 0 \\
\hline \multicolumn{7}{|l|}{ Overrepresented GO terms - diversifying selection } \\
\hline transmembrane transporter activity & $2.28 \mathrm{E}-02$ & 1.19E-02 & 4 & 23 & 4 & 18 \\
\hline transmembrane transport & $2.17 \mathrm{E}-02$ & $1.25 \mathrm{E}-02$ & 6 & 48 & 6 & 41 \\
\hline phospholipid metabolic process & 4.11E-02 & 4.20E-02 & 1 & 0 & 1 & 0 \\
\hline regulation of Wnt signaling pathway & $4.11 \mathrm{E}-02$ & 4.20E-02 & 1 & 0 & 1 & 0 \\
\hline lipid phosphatase activity & 4.11E-02 & 4.20E-02 & 1 & 0 & 1 & 0 \\
\hline proteolysis & $3.20 \mathrm{E}-02$ & $2.18 \mathrm{E}-02$ & 6 & 53 & 6 & 47 \\
\hline acetyl-CoA carboxylase activity & 4.11E-02 & 4.20E-02 & 1 & 0 & 1 & 0 \\
\hline pyruvate metabolic process & 1.67E-03 & 1.75E-03 & 2 & 0 & 2 & 0 \\
\hline acetyl-CoA carboxylase complex & 4.11E-02 & 4.20E-02 & 1 & 0 & 1 & 0 \\
\hline $\begin{array}{l}\text { NAD-dependent histone deacetylase activity } \\
\text { (H3-K14 specific) }\end{array}$ & 4.11E-02 & 4.20E-02 & 1 & 0 & 1 & 0 \\
\hline histone $\mathrm{H} 3$ deacetylation & 4.11E-02 & 4.20E-02 & 1 & 0 & 1 & 0 \\
\hline intracellular signal transduction & $1.51 \mathrm{E}-02$ & 4.27E-02 & 4 & 20 & 3 & 16 \\
\hline $\begin{array}{l}\text { transforming growth factor beta receptor } \\
\text { signaling pathway }\end{array}$ & 4.11E-02 & 4.20E-02 & 1 & 0 & 1 & 0 \\
\hline serine-type endopeptidase activity & 5.27E-03 & 4.56E-03 & 4 & 14 & 4 & 13 \\
\hline cytoskeleton & 4.98E-02 & 4.14E-02 & 2 & 7 & 2 & 6 \\
\hline septin complex & 4.11E-02 & 4.20E-02 & 1 & 0 & 1 & 0 \\
\hline complement activation & 4.11E-02 & 4.20E-02 & 1 & 0 & 1 & 0 \\
\hline nociceptin receptor activity & 4.11E-02 & $1.00 E+00$ & 1 & 0 & 0 & 0 \\
\hline calcium-mediated signaling & 4.11E-02 & 4.20E-02 & 1 & 0 & 1 & 0 \\
\hline glycerolipid metabolic process & $3.98 \mathrm{E}-02$ & 4.14E-02 & 2 & 6 & 2 & 6 \\
\hline kinesin complex & 4.11E-02 & 4.20E-02 & 1 & 0 & 1 & 0 \\
\hline kinesin binding & 4.11E-02 & 4.20E-02 & 1 & 0 & 1 & 0 \\
\hline lipid catabolic process & $4.88 \mathrm{E}-03$ & $5.10 \mathrm{E}-03$ & 2 & 1 & 2 & 1 \\
\hline $\begin{array}{l}\text { oxidoreductase activity, acting on paired } \\
\text { donors, with incorporation or reduction of } \\
\text { molecular oxygen }\end{array}$ & $2.25 \mathrm{E}-02$ & 1.93E-01 & 2 & 4 & 1 & 4 \\
\hline L-ascorbic acid binding & $1.54 \mathrm{E}-02$ & $1.58 \mathrm{E}-01$ & 2 & 3 & 1 & 3 \\
\hline lipoprotein lipase activity & $1.67 \mathrm{E}-03$ & $1.75 \mathrm{E}-03$ & 2 & 0 & 2 & 0 \\
\hline Lys48-specific deubiquitinase activity & 4.11E-02 & $1.00 \mathrm{E}+00$ & 1 & 0 & 0 & 0 \\
\hline synapse & 4.11E-02 & 4.20E-02 & 1 & 0 & 1 & 0 \\
\hline Sin3 complex & 4.11E-02 & 4.20E-02 & 1 & 0 & 1 & 0 \\
\hline sulfate assimilation & 4.11E-02 & 4.20E-02 & 1 & 0 & 1 & 0 \\
\hline
\end{tabular}


Table 1 Over- and underrepresented terms from a GO enrichment analysis. Cor = Coregonus, Salv = Salvelinus, OGs = orthogroups (Continued)

\begin{tabular}{|c|c|c|c|c|c|c|}
\hline GO category & $p$-value & $\begin{array}{l}p \text {-value } \\
(\text { Cor + Salv })\end{array}$ & $\begin{array}{l}\text { \# in OGs under } \\
\text { selection }\end{array}$ & $\begin{array}{l}\text { \# in reference } \\
\text { OGs }\end{array}$ & $\begin{array}{l}\text { \# in OGs } \\
\text { under selection } \\
\text { (Cor + Salv) }\end{array}$ & $\begin{array}{l}\text { \# in reference } \\
\text { OGs (Cor + Salv) }\end{array}$ \\
\hline adenylylsulfate kinase activity & 4.11E-02 & 4.20E-02 & 1 & 0 & 1 & 0 \\
\hline sulfate adenylyltransferase (ATP) activity & 4.11E-02 & 4.20E-02 & 1 & 0 & 1 & 0 \\
\hline glycogen metabolic process & 4.11E-02 & 4.20E-02 & 1 & 0 & 1 & 0 \\
\hline $\begin{array}{l}\text { antigen processing and presentation of } \\
\text { endogenous peptide antigen via MHC class I }\end{array}$ & 4.11E-02 & 4.20E-02 & 1 & 0 & 1 & 0 \\
\hline melatonin receptor activity & 4.11E-02 & $1.00 E+00$ & 1 & 0 & 0 & 0 \\
\hline 3-hydroxyisobutyrate dehydrogenase activity & 4.11E-02 & 4.20E-02 & 1 & 0 & 1 & 0 \\
\hline NAD binding & $1.54 \mathrm{E}-02$ & 1.61E-02 & 2 & 3 & 2 & 3 \\
\hline urea transmembrane transporter activity & 4.11E-02 & 4.20E-02 & 1 & 0 & 1 & 0 \\
\hline urea transmembrane transport & 4.11E-02 & 4.20E-02 & 1 & 0 & 1 & 0 \\
\hline 5-methylcytosine catabolic process & 4.11E-02 & 4.20E-02 & 1 & 0 & 1 & 0 \\
\hline methylcytosine dioxygenase activity & 4.11E-02 & 4.20E-02 & 1 & 0 & 1 & 0 \\
\hline DNA demethylation & 4.11E-02 & 4.20E-02 & 1 & 0 & 1 & 0 \\
\hline pyrroline-5-carboxylate reductase activity & 4.11E-02 & 4.20E-02 & 1 & 0 & 1 & 0 \\
\hline proline biosynthetic process & 4.11E-02 & 4.20E-02 & 1 & 0 & 1 & 0 \\
\hline $\begin{array}{l}\text { malate dehydrogenase (decarboxylating) } \\
(\mathrm{NAD+} \text { ) activity }\end{array}$ & 4.11E-02 & 4.20E-02 & 1 & 0 & 1 & 0 \\
\hline malate metabolic process & 4.11E-02 & 4.20E-02 & 1 & 0 & 1 & 0 \\
\hline proteasome regulatory particle assembly & 4.11E-02 & 4.20E-02 & 1 & 0 & 1 & 0 \\
\hline beta1-adrenergic receptor activity & 4.11E-02 & $1.00 \mathrm{E}+00$ & 1 & 0 & 0 & 0 \\
\hline $\begin{array}{l}\text { adenylate cyclase-activating G protein-coupled } \\
\text { receptor signaling pathway }\end{array}$ & 4.11E-02 & $1.00 E+00$ & 1 & 0 & 0 & 0 \\
\hline positive regulation of heart contraction & 4.11E-02 & $1.00 E+00$ & 1 & 0 & 0 & 0 \\
\hline adrenergic receptor signaling pathway & 4.11E-02 & $1.00 \mathrm{E}+00$ & 1 & 0 & 0 & 0 \\
\hline small ribosomal subunit & 4.11E-02 & 4.20E-02 & 1 & 0 & 1 & 0 \\
\hline 10-formyltetrahydrofolate catabolic process & 4.11E-02 & 4.20E-02 & 1 & 0 & 1 & 0 \\
\hline formyltetrahydrofolate dehydrogenase activity & 4.11E-02 & 4.20E-02 & 1 & 0 & 1 & 0 \\
\hline $\begin{array}{l}\text { hydroxymethyl-, formyl- and related transferase } \\
\text { activity }\end{array}$ & 4.11E-02 & 4.20E-02 & 1 & 0 & 1 & 0 \\
\hline regulation of neuroinflammatory response & 4.11E-02 & $1.00 \mathrm{E}+00$ & 1 & 0 & 0 & 0 \\
\hline hexosaminidase activity & 4.11E-02 & $1.00 \mathrm{E}+00$ & 1 & 0 & 0 & 0 \\
\hline junctional membrane complex & 4.11E-02 & 4.20E-02 & 1 & 0 & 1 & 0 \\
\hline \multicolumn{7}{|l|}{ Underrepresented GO terms - diversifying selection } \\
\hline DNA binding & 1.89E-02 & 3.46E-02 & 0 & 93 & 0 & 77 \\
\hline
\end{tabular}

(st8sia6)'), organelle function (2 of 12 orthogroups, e.g., 'sterile alpha motif domain-containing protein 9like $(s a m d 9 l)$ ), bone growth (1 of 12 orthogroups, 'cathepsin K precursor (ctsk)'), and immunity (1 of 12 orthogroups, 'furin-1-like (fur1)'), amongst others (Table 2). Again, this agrees with the more general GO term functions inferred using Blast2GO and associated tools (Fig. 6c - biological process GO terms, Table 2), such as neurotransmitter secretion, negative regulation of transcription, serine family amino acid metabolism, fatty acid biosynthesis, and carbohydrate metabolism.

\section{Discussion}

Our analyses of shared selection in the highly diversifying taxa Coregonus and Salvelinus identified genes and gene functions with deviating signatures of selection compared to five relatively less diversifying salmonid taxa and one non-salmonid species used as background. We identified more orthogroups under relaxed selection 
a

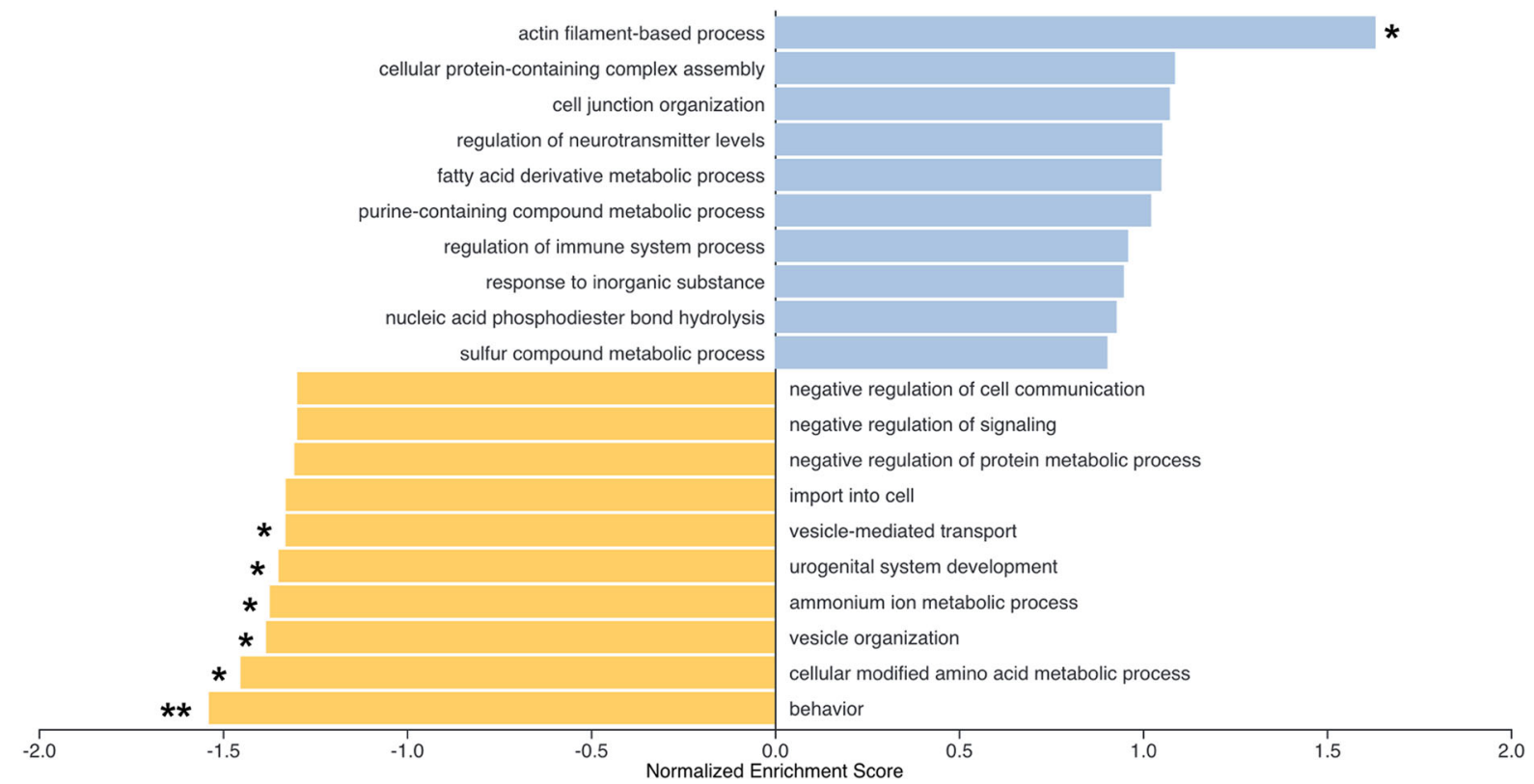

b

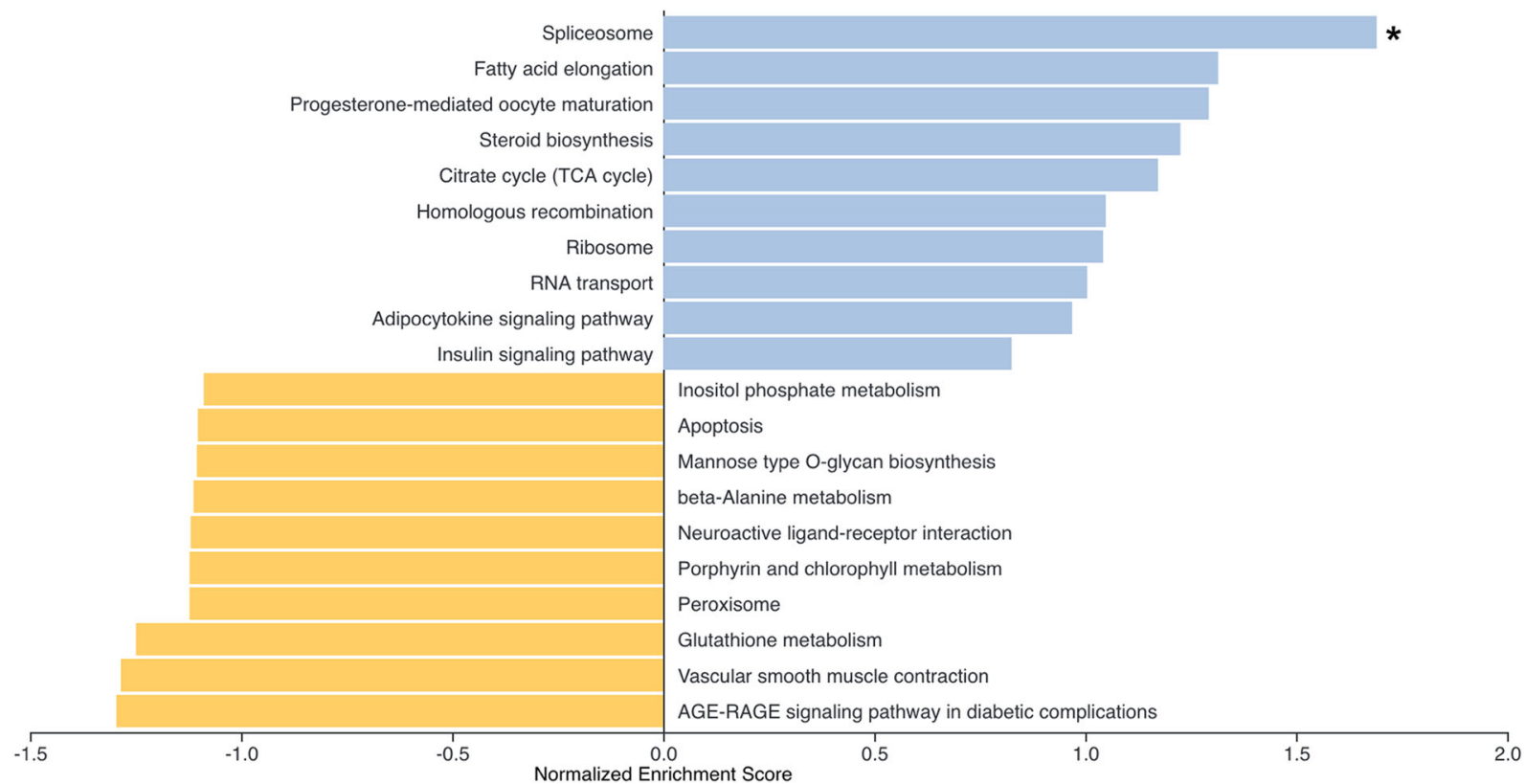

Fig. 5 Top ten enriched a biological process GO terms and $\mathbf{b}$ KEGG pathways based on a gene set enrichment analysis (GSEA) of all orthogroups in Coregonus and Salvelinus relative to non-diversifying taxa. Terms with negative scores (yellow) were inferred to be under relaxed selection and with positive scores (blue) under intensified selection. The normalised enrichment score is calculated from transformed $k$ values of each gene in a gene set. ${ }^{* *}$ indicates significant gene set enrichment after multiple correction $(F D R<0.2) ;{ }^{*}$ indicates significant gene set enrichment without multiple correction ( 0.05)

$(k$ value $<1)$ than under intensified selection $(k$ value $>$ 1) (Figs. 2 and 6, Additional file 1). Further, we identified a set of 92 orthogroups that reflect signals of diversifying selection, nine and 12 of which additionally experienced either relaxed or intensified selection, respectively (Fig. 6,
Table 2). We explored the underlying biological relevance and associated gene functions of these orthogroups under selection and observed trends of overrepresented GO terms in all three sets of orthogroups under selection (Fig. 4, Table 1). What is 


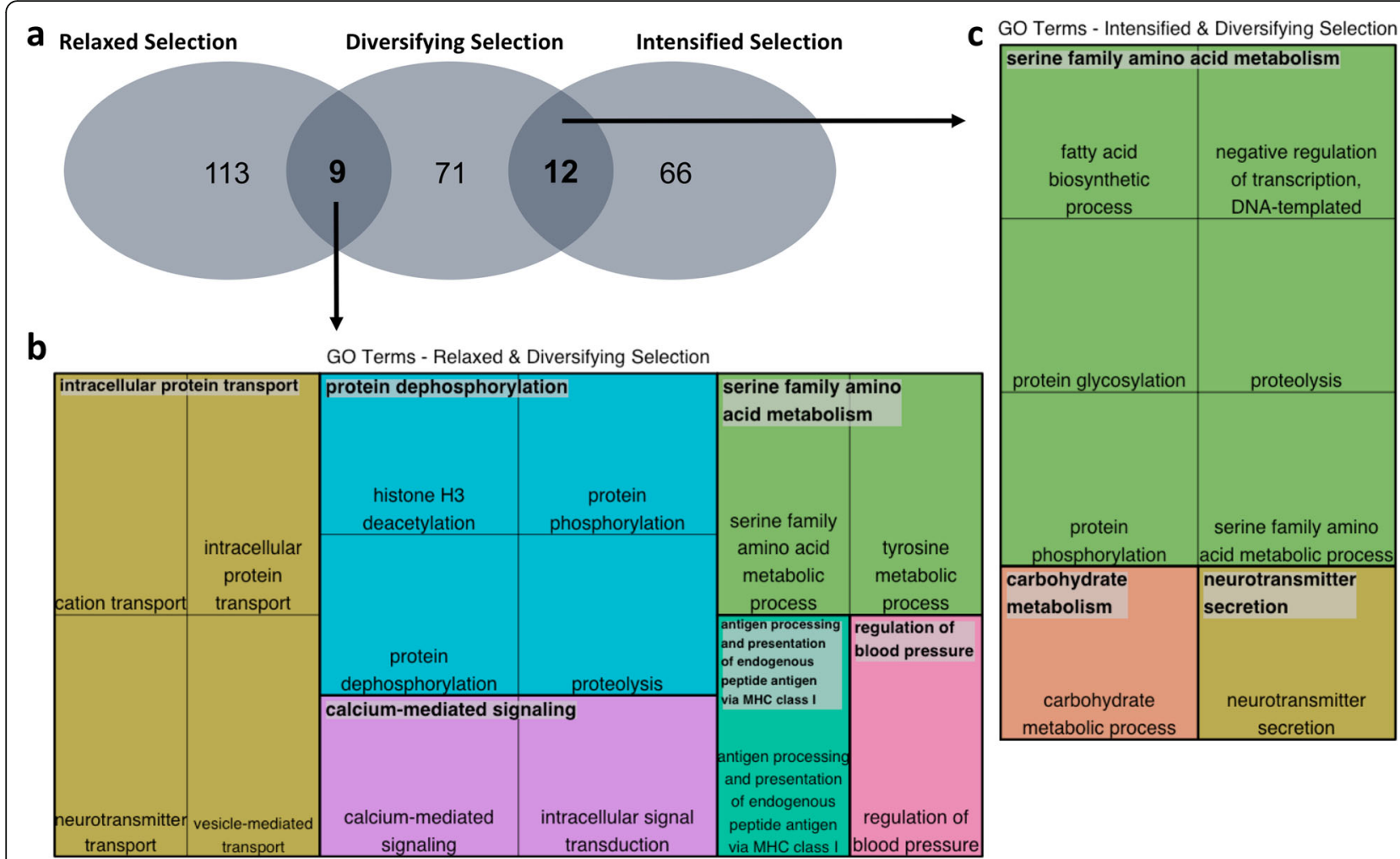

Fig. 6 Overlapping orthogroups under selection and biological process GO terms. a Overlap between orthogroups under relaxed and diversifying or intensified and diversifying selection in both Coregonus and Salvelinus. b Biological process GO terms of orthogroups under both relaxed and diversifying selection. c Biological process $\mathrm{GO}$ terms of orthogroups under both intensified and diversifying selection. In $\mathbf{b}$ and $\mathbf{c}$, rectangles are clustered into larger, coloured rectangles based on relationships among GO terms [70]

more, we identified $13 \mathrm{GO}$ terms with $k$ distributions deviating from the null expectation (Fig. 3).

In our functional analyses of the above selected orthogroups, genes in orthogroups under relaxed selection were found to be involved in processes such as transcriptional regulation, nervous system function, muscle and heart growth, lipid metabolism, and immunity, while orthogroups under intensified selection were found to be more often involved in signalling processes, actin filamentbased processes, RNA splicing, carbohydrate metabolism, transcriptional regulation as well as nervous system and bone development (Additional file 1). Orthogroups under relaxed selection were enriched for 'serine family amino acid metabolism' while those under intensified selection tended to be enriched more strongly for various signalling pathways (Fig. 4, Table 1). 'Regulation of transcription' was overrepresented in orthogroups under both relaxed and intensified selection. Orthogroups with signals of diversifying selection showed a clear trend towards enrichment for signal transduction, transmembrane transport, proteolysis, lipid metabolism, and pyruvate metabolism GO terms (Fig. 4, Table 1) and often contained genes involved in transcriptional regulation, development, lipid metabolism, and immunity (orthogroups under diversifying selection,
Additional file 2, including associated GO terms). Therefore, our results imply different intensities of selection on different gene functions and processes.

\section{Selection parameter distribution - shift to relaxation?}

Our results agree with the repeated finding of a relaxation of selective constraint in rapidly diverging taxa $[18,19,72,73]$. One possible cause behind this observation could be an increase of ecological opportunity due to the emergence of new habitat and a decrease in interspecies competition, for example in caves [72] or within postglacial lakes [74-79]. The adaptation to life in lakes, before or in the course of (repeated) postglacial colonisations, such as in charr and whitefish, could be such an ecological opportunity, entailing a release of selective pressures on a substantial number of genes.

From the above results we can also conclude that relaxation of selective constraints does not affect the whole set of protein-coding genes to the same degree. Purifying selection and selective constraint still affect a large number of genes with functions that ensure an organism's integrity, survival, and reproduction (see orthogroups under intensified selection) [80]. However, in charr and whitefish we observed a larger number of orthogroups 
Table 2 Orthogroups (OGs) with signals of relaxed and diversifying selection or intensified and diversifying selection. $k$ values are selection parameter values (exponent of $d N / d S$ ratio, i.e., $k$ in $\omega^{k}$ ). FDR values are from Benjamini-Hochberg FDR correction. Orthogroups shown are present in both Coregonus and Salvelinus. For aBSREL, $p$-values after correction for multiple testing (FDR $<0.10)$ are shown

\begin{tabular}{|c|c|c|c|c|c|c|}
\hline OG ID & Gene description & $\begin{array}{l}\text { GO terms (obtained with } \\
\text { Blast2GO) }\end{array}$ & $\begin{array}{l}\text { Additional information (manually } \\
\text { derived from UniProt/Swiss-Prot) }\end{array}$ & $\begin{array}{l}\text { FDR } \\
(\text { RELAX) }\end{array}$ & $p(\mathrm{aBSREL})$ & k \\
\hline \multicolumn{7}{|c|}{ Relaxed \& diversifying selection } \\
\hline OG0001544 & $\begin{array}{l}\text { adaptor-related protein } \\
\text { complex 1, mu } 2 \text { subunit } \\
\text { (ap1m2) }\end{array}$ & $\begin{array}{l}\text { intracellular protein transport; } \\
\text { vesicle-mediated transport; } \\
\text { clathrin adaptor complex }\end{array}$ & $\begin{array}{l}\text { antigen processing and } \\
\text { presentation of exogenous } \\
\text { peptide antigen via } \mathrm{MHC} \text { class II, } \\
\text { regulation of defence response } \\
\text { to virus by virus }\end{array}$ & 0.000 & $1.019 \mathrm{E}-05$ & 0.04471 \\
\hline OG0002461 & $\begin{array}{l}\text { protein kinase C epsilon } \\
\text { type-like (LOC106589411) }\end{array}$ & $\begin{array}{l}\text { protein serine/threonine kinase } \\
\text { activity; ATP binding; protein } \\
\text { phosphorylation; serine family } \\
\text { amino acid metabolic process; } \\
\text { intracellular signal transduction }\end{array}$ & $\begin{array}{l}\text { cell adhesion, motility, and } \\
\text { cell cycle, neuron growth, ion } \\
\text { channel regulation, immune } \\
\text { response, signal transduction }\end{array}$ & $6.825 \mathrm{E}-08$ & $6.478 \mathrm{E}-06$ & 0.07476 \\
\hline OG0003986 & $\begin{array}{l}\text { solute carrier family } 6 \\
\text { (neurotransmitter } \\
\text { transporter), member } 8 \\
\text { (slc6a8) }\end{array}$ & $\begin{array}{l}\text { neurotransmitter:sodium } \\
\text { symporter activity; cation } \\
\text { transport; neurotransmitter } \\
\text { transport; integral component } \\
\text { of membrane }\end{array}$ & $\begin{array}{l}\text { muscle contraction, required for } \\
\text { the uptake of creatine in muscles } \\
\text { and brain }\end{array}$ & 7.970E-05 & $3.046 \mathrm{E}-12$ & 0.05603 \\
\hline OG0000840 & $\begin{array}{l}\text { probable histone } \\
\text { deacetylase 1-B (hda1b) }\end{array}$ & $\begin{array}{l}\text { nucleus; NAD-dependent histone } \\
\text { deacetylase activity (H3-K14 } \\
\text { specific); metal ion binding; } \\
\text { histone H3 deacetylation }\end{array}$ & $\begin{array}{l}\text { transcriptional regulation, cell } \\
\text { cycle progression, developmental } \\
\text { events }\end{array}$ & 0.0002432 & $1.952 \mathrm{E}-06$ & $2.398 \mathrm{E}-12$ \\
\hline OG0005675 & $\begin{array}{l}\text { E3 ubiquitin-protein } \\
\text { ligase RNF128-like } \\
\text { (LOC110537269), } \\
\text { transcript variant X1 }\end{array}$ & - & $\begin{array}{l}\text { interleukin regulation, T-cell } \\
\text { control, functions in the } \\
\text { patterning of the dorsal } \\
\text { ectoderm, sensitises ectoderm } \\
\text { to respond to neural-inducing } \\
\text { signals }\end{array}$ & 0.0002432 & $1.282 \mathrm{E}-08$ & 0.5357 \\
\hline OG0004474 & $\begin{array}{l}\text { endoplasmic reticulum } \\
\text { aminopeptidase 1-like } \\
\text { (LOC106570844) }\end{array}$ & $\begin{array}{l}\text { endoplasmic reticulum } \\
\text { membrane; proteolysis; } \\
\text { regulation of blood pressure; } \\
\text { zinc ion binding; antigen } \\
\text { processing and presentation } \\
\text { of endogenous peptide } \\
\text { antigen via MHC class l; } \\
\text { metalloaminopeptidase } \\
\text { activity }\end{array}$ & $\begin{array}{l}\text { regulation of innate immune } \\
\text { response, response to bacterium, } \\
\text { angiogenesis, regulation of blood } \\
\text { pressure, fat cell differentiation }\end{array}$ & 0.01917 & $2.008 \mathrm{E}-05$ & 0.1651 \\
\hline OG0000651 & $\begin{array}{l}\text { tyrosine-protein } \\
\text { phosphatase non- } \\
\text { receptor type 6-like } \\
\text { (LOC106576571), } \\
\text { transcript variant X3 }\end{array}$ & $\begin{array}{l}\text { protein tyrosine } \\
\text { phosphatase activity; } \\
\text { protein dephosphorylation; } \\
\text { tyrosine metabolic process }\end{array}$ & $\begin{array}{l}\text { haematopoiesis, various } \\
\text { immune functions }\end{array}$ & 0.03186 & $1.547 \mathrm{E}-06$ & 0.3844 \\
\hline OG0002139 & $\begin{array}{l}\text { calcipressin-2-like } \\
\text { (LOC109900784), } \\
\text { transcript variant X1 }\end{array}$ & $\begin{array}{l}\text { nucleic acid binding; } \\
\text { calcium-mediated signaling }\end{array}$ & $\begin{array}{l}\text { regulation of calcineurin-NFAT } \\
\text { signalling cascade, calcium- } \\
\text { mediated signalling, potential } \\
\text { role in central nervous } \\
\text { development }\end{array}$ & 0.06629 & $2.753 \mathrm{E}-11$ & 0.6235 \\
\hline OG0014882 & $\begin{array}{l}\text { junctophilin-2 } \\
\text { (LOC111976619) }\end{array}$ & junctional membrane complex & $\begin{array}{l}\text { calcium ion homeostasis, } \\
\text { regulation of cardiac muscle } \\
\text { tissue development }\end{array}$ & 0.08725 & $3.460 \mathrm{E}-09$ & 0.6595 \\
\hline \multicolumn{7}{|c|}{ Intensified \& diversifying selection } \\
\hline OG0000798 & $\begin{array}{l}\text { acetyl-CoA carboxylase } \\
\text { beta (acacb), transcript } \\
\text { variant X3 }\end{array}$ & $\begin{array}{l}\text { acetyl-CoA carboxylase activity; } \\
\text { ATP binding; pyruvate metabolic } \\
\text { process; fatty acid biosynthetic } \\
\text { process; acetyl-CoA carboxylase } \\
\text { complex; metal ion binding }\end{array}$ & $\begin{array}{l}\text { regulation of cholesterol } \\
\text { biosynthetic process, acetyl-CoA } \\
\text { metabolic process }\end{array}$ & 0.000 & 0.000 & 4.683 \\
\hline OG0011982 & $\begin{array}{l}\text { synapsin-3 } \\
(\text { LOC } 111950806)\end{array}$ & $\begin{array}{l}\text { ATP binding; neurotransmitter } \\
\text { secretion; synaptic vesicle }\end{array}$ & $\begin{array}{l}\text { neurotransmitter secretion, } \\
\text { regulation of synaptic } \\
\text { transmission, GABAergic }\end{array}$ & 0.0001606 & 0.000 & 8.330 \\
\hline OG0004141 & clone Contig3164 & proteolysis; cysteine-type & osteoclastic bone resorption, & 0.0002432 & $3.664 \mathrm{E}-14$ & 7.769 \\
\hline
\end{tabular}


Table 2 Orthogroups (OGs) with signals of relaxed and diversifying selection or intensified and diversifying selection. $k$ values are selection parameter values (exponent of $d N / d S$ ratio, i.e., $k$ in $\omega^{k}$ ). FDR values are from Benjamini-Hochberg FDR correction. Orthogroups shown are present in both Coregonus and Salvelinus. For aBSREL, $p$-values after correction for multiple testing (FDR $<0.10)$ are shown (Continued)

\begin{tabular}{|c|c|c|c|c|c|c|}
\hline OG ID & Gene description & $\begin{array}{l}\text { GO terms (obtained with } \\
\text { Blast2GO) }\end{array}$ & $\begin{array}{l}\text { Additional information (manually } \\
\text { derived from UniProt/Swiss-Prot) }\end{array}$ & $\begin{array}{l}\text { FDR } \\
\text { (RELAX) }\end{array}$ & $p$ (aBSREL) & k \\
\hline & cathepsin $\mathrm{K}$ precursor & peptidase activity & $\begin{array}{l}\text { bone remodelling, collagen } \\
\text { metabolic process }\end{array}$ & & & \\
\hline OG0013215 & $\begin{array}{l}\text { lysine-specific } \\
\text { demethylase 4B-like } \\
\text { (LOC106574093), } \\
\text { transcript variant X3 }\end{array}$ & $\begin{array}{l}\text { histone H3-K36 demethylation; } \\
\text { histone H3-K9 demethylation } \\
\text { (manually derived from UniProt/ } \\
\text { Swiss-Prot) }\end{array}$ & $\begin{array}{l}\text { histone } \mathrm{H} 3-\mathrm{K} 36 \text { demethylation, } \\
\text { histone } \mathrm{H} 3-\mathrm{K} 9 \text { demethylation, } \\
\text { negative regulation of } \\
\text { transcription }\end{array}$ & 0.003614 & 3.970E-12 & 10.28 \\
\hline OG0003822 & $\begin{array}{l}\text { sterile alpha motif } \\
\text { domain-containing } \\
\text { protein 9-like } \\
\text { (LOC109881303), } \\
\text { transcript variant X2 }\end{array}$ & protein binding & endosomal vesicle fusion & 0.006278 & 9.533E-06 & 1.603 \\
\hline OG0007378 & $\begin{array}{l}\text { alpha-2,8-sialyltransferase } \\
\text { 8F-like (LOC106588277), } \\
\text { transcript variant X2 }\end{array}$ & $\begin{array}{l}\text { protein glycosylation; } \\
\text { sialyltransferase activity }\end{array}$ & $\begin{array}{l}\text { carbohydrate biosynthetic } \\
\text { process, glycolipid metabolic } \\
\text { process, ganglioside biosynthetic } \\
\text { process, (modulation of cell } \\
\text { signal transduction, maybe } \\
\text { involved in function of nervous } \\
\text { system) }\end{array}$ & 0.008768 & $\begin{array}{l}2.090 \mathrm{E}-11 \\
9.549 \mathrm{E}-08\end{array}$ & 1.9625 \\
\hline OG0003820 & $\begin{array}{l}\text { paired amphipathic } \\
\text { helix protein Sin3a-like } \\
\text { (LOC106587652), } \\
\text { transcript variant X3 }\end{array}$ & $\begin{array}{l}\text { transcription corepressor } \\
\text { activity; transcription factor } \\
\text { complex; negative regulation } \\
\text { of transcription }\end{array}$ & $\begin{array}{l}\text { negative regulation of } \\
\text { transcription, regulation of } \\
\text { lipid metabolic process, } \\
\text { regulation of circadian } \\
\text { rhythms }\end{array}$ & 0.01812 & $5.292 \mathrm{E}-09$ & 1.450 \\
\hline OG0004629 & $\begin{array}{l}\text { furin-1-like } \\
\text { (LOC106587394), } \\
\text { transcript variant X2 }\end{array}$ & $\begin{array}{l}\text { serine-type endopeptidase } \\
\text { activity; proteolysis }\end{array}$ & $\begin{array}{l}\text { involved in microbial infection, } \\
\text { regulation of endopeptidase } \\
\text { activity, TGF-beta-1 activation }\end{array}$ & 0.01896 & 0.000 & 19.99 \\
\hline OG0010786 & $\begin{array}{l}\text { serine/threonine-protein } \\
\text { kinase } 6 \text { (stk6) }\end{array}$ & $\begin{array}{l}\text { protein serine/threonine kinase } \\
\text { activity; ATP binding; protein } \\
\text { phosphorylation; serine family } \\
\text { amino acid metabolic process }\end{array}$ & $\begin{array}{l}\text { activation of protein kinase } \\
\text { activity, regulation of } \\
\text { transcription }\end{array}$ & 0.01972 & 0.000 & 6.183 \\
\hline OG0012826 & $\begin{array}{l}\text { beta-1,4- } \\
\text { galactosyltransferase } \\
\text { 6-like (LOC106560385), } \\
\text { transcript variant X1 }\end{array}$ & $\begin{array}{l}\text { carbohydrate metabolic process; } \\
\text { transferase activity }\end{array}$ & $\begin{array}{l}\text { neuronal maturation, axonal } \\
\text { and myelin formation, } \\
\text { lactosylceramide biosynthetic } \\
\text { process, carbohydrate metabolic } \\
\text { process }\end{array}$ & 0.03015 & $1.221 \mathrm{E}-12$ & 6.768 \\
\hline OG0010420 & $\begin{array}{l}\text { torsin-1A-interacting } \\
\text { protein } 2 \text { (LOC111975742) }\end{array}$ & $\begin{array}{l}\text { endoplasmic reticulum } \\
\text { organization; positive } \\
\text { regulation of ATPase } \\
\text { activity; protein localization } \\
\text { to nuclear envelope (manually } \\
\text { derived from UniProt/Swiss-Prot) }\end{array}$ & $\begin{array}{l}\text { endoplasmic reticulum integrity, } \\
\text { positive regulation of ATPase } \\
\text { activity }\end{array}$ & 0.03800 & 0.000 & 1.870 \\
\hline OG0011057 & $\begin{array}{l}\text { receptor-interacting } \\
\text { serine/threonine- } \\
\text { protein kinase 4-like } \\
\text { (LOC106567956) }\end{array}$ & $\begin{array}{l}\text { protein kinase activity; protein } \\
\text { binding; ATP binding; protein } \\
\text { phosphorylation }\end{array}$ & $\begin{array}{l}\text { positive regulation of NF-kappaB } \\
\text { transcription factor activity }\end{array}$ & 0.08386 & $5.012 \mathrm{E}-06$ & 3.356 \\
\hline
\end{tabular}

under relaxed selection that therefore escaped the effects of purifying selection.

The observation of largely different GO terms in orthogroups under relaxed selection or intensified selection agrees with a large number of studies that demonstrated different selective pressures on different gene functions [81, 82]. Various functional and structural constraints govern the substitution patterns within genes. For example, amino acid alterations in some groups of proteins can easily make them dysfunctional [81], while other kinds of proteins are under less constraint to vary [83] or are even beneficial to fitness when being different from the majority of the population (i.e., negative frequency-dependent or balanced selection) [84-86]. The observation of intensified selection on DNA repair in our dataset and the overrepresented GO terms related to oxidative stress and apoptotic signalling, for example, could highlight the importance of an effective response to environmental stress in rapidly 
diversifying taxa. Also, carbohydrate and ribosome-related processes seem to be under strong selective constraint. On the other hand, relaxation of selective constraint on ATPase activity and proton transmembrane transport as well as various lipid metabolism genes, amongst others, could have led to the physiological variety observed in extant highly diversifying salmonids.

\section{Gene functions under relaxed selection in Coregonus and Salvelinus}

Many of the orthogroups under parallel relaxed selection, i.e. those that experienced a release of selective constraint in Coregonus and Salvelinus, have been linked to the divergence of these species along the benthic-limnetic axis within lakes found in earlier research [74, 87-91]. Examples include the 'peripherin-2-like' gene, which has been implicated in visual adaptation in S. namaycush in Lake Superior [92] and the 'natterin-3-like' gene, which plays a putative role as an immunopeptide and experienced pronounced expression divergence in Icelandic $S$. alpinus ecomorphs [93, 94]. Based on our analyses, we propose these are linked to the evolution of novel phenotypes and the variation in feeding and swimming behaviour, energy storage, and the release of competition in postglacial lakes (behaviour, muscle and heart growth, locomotion, lipid metabolism, nervous system function) as well as a release or change of parasite and pathogen burden (immunity genes) in postglacial lakes [95]. This implies changes in selective pressure on these gene functions as a consequence of shifts in the ecology of highly diversifying salmonids.

\section{Gene functions under intensified selection in Coregonus and Salvelinus}

Genes under intensified selection are expected to have a strong role for survival and reproduction (i.e., fitness) of organisms [49]. We found the orthogroups under intensified selection showed a high number of processes associated with key metabolic pathways (mainly of carbohydrates and lipids), signal transduction, and regulation of gene expression and the immune system, indicating strong selective pressures on these processes and functions in Coregonus and Salvelinus. The occurrence of immunity genes under intensified selection in our results would rather imply changing parasite and pathogen regimes rather than a complete release of pathogen burden [96]. Interestingly, our results also indicated strong selective pressures on actin filament-based processes and RNA splicing as well as protein modification, which are functions with a crucial role in development [96-99]. Among these genes, some were found to be directly linked to differences in bone development [e.g., 'paired like homeodomain 1' and 'cathepsin K precursor') [100-102]. We speculate that these developmental process genes may be relevant in the extreme morphological diversity that can be found among closely related Coregonus species and particularly Salvelinus species [90, 100-104].

\section{Gene functions under diversifying selection in Coregonus and Salvelinus}

Diversifying (i.e., positive) selection on genes, which in this context means that selective pressure favours amino acid polymorphism in a particular gene, affected 92 orthogroups in Coregonus and Salvelinus relative to the non-diversifying taxa. The enrichment of lipid, glycerolipid, and pyruvate metabolism genes is a strong indication that these metabolic processes experienced diversification/positive selection in Coregonus and Salvelinus, which could imply a role in ecological diversification and habitat shifts across the depth axis in lakes. Lipid metabolism has been commonly found to be under diversifying selection in freshwater fishes, such as sticklebacks [105] and salmonids [89, 106]. Also, diversifying/positive selection on transmembrane transport, for example, has been shown repeatedly in teleost fish adapting to the freshwater environment, such as sculpin and alewife, putatively because of its role in osmoregulation [107, 108].

\section{Overlap between selection types}

A combination of approaches, such as aBSREL and RELAX as we apply here, has been shown to reliably infer genes under selection and is also able to appropriately distinguish between positive selection and relaxed selection, which are hard to disentangle using single conventional methods [109-111]. This is particularly important since it has been known for some time that the number of false positives in tests for positive or diversifying selection can be substantial [112-115]. Overlapping sets of orthogroups under selection inferred with different analytical approaches, as we resolve here, can therefore implicate consistent, strong, and specific signals of selection. For example, orthogroups with signals of relaxed and diversifying selection likely experienced a release of purifying selection, with the potential for evolutionary diversification $[19,49,116]$.

One of the orthogroups with the most extreme signal of relaxed and diversifying selection was 'adaptor-related protein complex 1, mu 2 subunit (ap1m2)' (Table 2). Apart from a role in endothelial and intestinal immunity [117], ap1m2 plays a central role in the development of endoderm-derived organs (e.g., stomach and intestine) in zebrafish [118]. Given the differing parasite pressure [119, 120] and the diversity of trophic niches in lake systems $[121,122]$, genes under reduced selective constraint could play a crucial role in phenotypic diversity in the often lake-dwelling Salvelinus and Coregonus. Another gene showing a strong signal of relaxed and diversifying selection was 'solute carrier family 6 (neurotransmitter transporter), member 8 (slc6a8)', which regulates creatine 
uptake in tissues with high energy demands such as muscle and brain tissue [122-124]. Recent research on $C$. mykiss and C. maraena found two copies of this gene, which were either mainly expressed in muscle or kidney tissue, with the strongest expression overall in muscle [125]. Changes of selection regimes on these functions are expected, given that Salvelinus and Coregonus experienced a diversification in trophic and swimming behaviour, ecology, and life history (e.g., Salvelinus and Coregonus can be free swimming, lake bottom dwelling, or restricted to the littoral zone of lakes). Our analyses independently corroborate an important functional role of this gene in highly diversifying salmonids.

Orthogroups showing both intensified and diversifying selection likely constitute targets of "true" positive selection rather than relaxed selection misinterpreted as positive selection. Genes in these orthogroups are thus under stronger selective pressure and are potentially of higher importance to survival and overall fitness [49] in our analyses of Coregonus and Salvelinus. Two of the most strongly significant orthogroups under intensified and diversifying selection involved the genes 'synapsin-3 (syn3)' and 'cathepsin K precursor (ctsk)'. syn3 is involved in neurotransmitter secretion [126] and plays a crucial role in early neuronal differentiation and in neuronal progenitor cell development in fish [127] as well as in mammals [128]. ctsk, encoding a collagenase, has an important function in bone modelling and remodelling as well as bone homeostasis in vertebrates [129, 130] and is differentially expressed in S. alpinus [102]. Both syn 3 and ctsk are among the genes experiencing the highest selective pressure in Coregonus and Salvelinus as compared to other salmonid taxa, which is an indication of their importance for survival in these two genera and warrants further research.

Not only specific genes but also the gene functions associated with orthogroups under selection were found to have biological relevance. Changes in heart and muscle function, immunity genes, lipid metabolism, and transcriptional regulation have previously been implicated in diversification in salmonids [81, 131-136], cichlids [33], ants [83], and tonguefishes [84]. Therefore, our findings from molecular evolution agree with evidence from ecomorphological divergence of Coregonus and Salvelinus of immune response, feeding, metabolism, and locomotion $[46,137,138]$.

\section{Whole-genome duplication and potential effects on relaxed selection}

Multiple lines of evidence suggest that whole-genome duplication (WGD) events give rise to vast amounts of genetic material that can subsequently experience elevated substitution rates and relaxation of selective constraints [139-141]. The lineage ancestral to salmonids experienced a WGD around 80-103 Mya [67-69]. It has been speculated that this may have contributed to new phenotypic innovations and to the ecological success of salmonids [67, 142-145], although a contribution to diversification rates is contentious $[67,146]$. While conventional approaches for the detection of positive and relaxed selection based on orthologues in salmonids would potentially be biased in the case of duplicated and non-rediploidised genes, approaches based on orthogroups, as we applied here, alleviate this issue by explicitly comparing selection shared in Coregonus and Salvelinus relative to all other salmonid lineages [58]. This approach circumvents the difficulty of inferring orthologues in taxa that experienced multiple duplications and unequal rediploidisation.

While genes duplicated during the salmonid WGD might be more susceptible to relaxed selection [49, 147], charr and whitefish are in different subfamilies and not sister genera (Fig. 1) [41, 148]. Therefore, based on evolutionary relationships, they should have rediploidisation patterns more similar to their sister genera than to each other and there is no reason to suspect unequal rediploidisation among genera to be the cause of the relaxed selection signals we identified in Coregonus and Salvelinus. It is more likely that these two genera experienced similar selective pressures due to common diversification patterns, environmental conditions, or other common ecological or life history traits. Our results from molecular evolution analyses reflect these shared ecological and evolutionary patterns. However, recent results on gene expression in duplicated genes of salmonids have shown that relaxed selection often occurs in downregulated gene duplicates [149], which might be associated with dosage balance effects [150, 151]. Therefore, future research should ideally analyse selection in concert with gene expression and duplication.

\section{Conclusions}

Our findings suggest that there is some evidence for a parallel relaxation of selective constraint in the repeatedly diversifying salmonid lineages Coregonus and Salvelinus compared to all other salmonid lineages, which are known to be less diversifying. Genes in orthogroups under relaxed selection are involved in functions with a potential role in the rapid diversification and ecological adaptation that can be observed in wild populations of Coregonus and Salvelinus and experienced a release of selective pressure, including genes involved in behaviour, muscle function, and infection. On the other hand, orthogroups under intensified selection, such as various signal transduction and regulatory genes, are under stronger selective pressure and are consequently expected to have higher fitness effects in charr and whitefish compared to other salmonids. An independent 
analysis of orthogroups under diversifying selection showed an enrichment for signal transduction genes and GO terms of various metabolic processes, while genes involved in DNA binding were underrepresented. Orthogroups with both signals of relaxed and diversifying or intensified and diversifying selection can give further hints as to what selective processes govern the evolution of these genes and are also important candidates for gene sets under particularly weak (relaxed) selective pressure (while still experiencing molecular diversification), or strong (intensified) selective pressure. The discovered orthogroups under selection are a valuable resource for further investigations into the importance of certain genes for rapid diversification in salmonids and beyond.

\section{Material \& methods}

\section{Taxonomic dataset}

All analyses were performed on RNAseq transcriptome datasets drawn from a search in NCBI and a literature search in Google Scholar (keywords: each salmonid genus and species, in combination with "transcriptome" and/or "RNAseq" or "RNA-seq") to identify all available studies. The final dataset represents one transcriptome per species of transcriptomes of all available taxa as of October 2016, with additional in-house data for Salmo salar, Salmo trutta, Salvelinus alpinus, and Coregonus lavaretus that was published at a later point [88]. We aimed to retain transcriptomes with a maximal number of overlapping transcripts across salmonids, which consisted of nine species representing all five major genera (Coregonus, Oncorhynchus, Salmo, Salvelinus, and Thymallus), and the closest relative as outgroup (Esox lucius) (Fig. 1, Table 3). The selected transcriptome assemblies were all derived from several tissues so as to obtain almost complete sets of transcripts (see references in Table 3). The topology for foreground branch definitions is based on the phylogenetic tree shown in Fig. 1, which was obtained using maximum-likelihood tree estimation based on a preliminary set of 1564 orthologues (derived from the same set of transcriptomes; orthologue alignments available upon request) and in agreement with the current understanding of salmonid phylogenetic relationships [41, 148]. From the full dataset, ranging from 59.0 to $209.2 \mathrm{Mbp}$ per transcriptome (Table 3), we obtained 2702 sequences of orthogroups (mean number of orthologues per orthogroup: 4.77; mean length $1783 \mathrm{bp}$; range 607-14,743 bp; total 4.82 Mbp). Using a Blast2GO [156] analysis (for details see below), we confirmed that the dataset was not enriched for any particular GO categories (Fisher's Exact Test: all $p>0.10$ ) and therefore not a biased representation of transcripts.

\section{Orthogroup and OG tree inference}

The longest open reading frames (ORFs) in the transcriptome sequences of the ten species (excluding mitochondrial genes due to different genetic codes that could bias the selection analyses) and, additionally, protein-coding genes of the salmon genome (NCBI RefSeq assembly accession: GCF_000233375.1) [68] were determined using TransDecoder v2.1 (https:/github. com/TransDecoder/TransDecoder). Next, the standard mode of OrthoFinder v2.2.3 [157], which uses a five-step algorithm that mitigates gene length bias and other biases introduced by other methods, was used to infer orthogroups from the TransDecoder ORFs. Based on the OrthoFinder orthogroup assignments, gene sequences were then extracted from the whole transcriptomes with custom Python scripts (Python v3.6.5) if they were also present in the protein-coding sequences of the Atlantic salmon genome v2 (NCBI RefSeq assembly accession: GCF_000233375.1) [68] to exclude spurious transcripts. To further minimise the number of spurious transcripts retained, only orthogroups with a higher or equal number (but not larger than four) of orthologous genes in the focal species compared to the salmon genome were included to avoid inclusion of collapsed orthologues (i.e., multiple genes that were merged into one during assembly, which seems to be a common issue in the salmonid transcriptomes published so far according to in-house results and A. Yurchenko and M. Carruthers, personal communication). Orthologues were then combined into orthogroups using custom Python scripts if they matched the same gene sets in the salmon reference genome. $\mathrm{Du}$ plicated orthogroups, inferred as multiple occurrences of the same orthologue sets, orthogroups with a number of sequences exceeding 80 , which could include an inflated number of duplicates, or lower than seven, to avoid orthogroups unrepresentative of the whole dataset, and those with fewer than seven taxa were removed with custom Python scripts. The longest ORFs with a minimum length of $200 \mathrm{bp}$ were then extracted from this orthogroup set with get_orfs_or_cdss.py (https://github.com/peterjc/ pico_galaxy/blob/master/tools/get_orfs_or_cdss/get_orfs_ or_cdss.py). A codon-based alignment was then produced using PRANK v.140603 [158]. The aligned orthogroups were then trimmed and filtered with trimAl (-resoverlap 1.0, -seqoverlap 0.38 -noallgaps) [159], optimising both alignment quality and the number of retained alignments. Using the final set of orthogroups as input for FastME [160], we then constructed phylogenies of each orthogroup separately for use in the selection analyses below. The number of retained genes per orthogroup was calculated using custom BASH (BASH v3.2) and Python scripts.

\section{Selection analyses}

Foreground branches for subsequent selection analyses were labelled using a custom $R$ script $(R$ v3.5.1) and the $R$ package ape [161-163]. All selection analyses were 
Table 3 Sources of transcriptome assemblies used in this study

\begin{tabular}{|c|c|c|c|c|}
\hline \multicolumn{5}{|l|}{ Transcriptomes } \\
\hline Species & Source/Accession no. & Size $[M b p]$ & Transcript count & Reference \\
\hline $\begin{array}{l}\text { Coregonus clupeaformis } \\
\text { (lake whitefish) }\end{array}$ & PhyloFish (phylofish.sigenae.org, last access: 05.10.2016) & 158.5 & 66,996 & [152] \\
\hline Esox lucius (northern pike) & GATF00000000.1 (NCBI GenBank) & 188.7 & 55,424 & [153] \\
\hline $\begin{array}{l}\text { Oncorhynchus mykiss } \\
\text { (rainbow trout) }\end{array}$ & $\begin{array}{l}\text { Salmon Transcriptome website (salmon.cgrb.oregonstate.edu, } \\
\text { last access: } 05.10 .2016 \text { ) }\end{array}$ & 209.2 & 130,599 & [154] \\
\hline $\begin{array}{l}\text { Oncorhynchus tshawytscha } \\
\text { (Chinook salmon) }\end{array}$ & GSE59756 (NCBI Gene Expression Omnibus) & 159.8 & 183,740 & [155] \\
\hline $\begin{array}{l}\text { Salvelinus fontinalis } \\
\text { (brook trout) }\end{array}$ & PhyloFish (phylofish.sigenae.org, last access: 05.10.2016) & 166.0 & 69,441 & [152] \\
\hline $\begin{array}{l}\text { Thymallus thymallus } \\
\text { (European grayling) }\end{array}$ & PhyloFish (phylofish.sigenae.org, last access: 05.10.2016) & 160.9 & 67,157 & [152] \\
\hline $\begin{array}{l}\text { Coregonus lavaretus } \\
\text { (European whitefish) }\end{array}$ & $\begin{array}{l}\text { earlier version of GGAO00000000.1 (NCBI GenBank; made } \\
\text { accessible upon request) }\end{array}$ & 59.0 & 44,348 & [88] \\
\hline $\begin{array}{l}\text { Salvelinus alpinus } \\
\text { (Arctic charr) }\end{array}$ & $\begin{array}{l}\text { earlier version of GGAP00000000.1 (NCBI GenBank; made } \\
\text { accessible upon request) }\end{array}$ & 59.5 & 44,354 & [88] \\
\hline $\begin{array}{l}\text { Salmo salar } \\
\text { (Atlantic salmon) }\end{array}$ & $\begin{array}{l}\text { earlier version of GGAQ00000000.1 (NCBI GenBank; made } \\
\text { accessible upon request) }\end{array}$ & 63.0 & 44,554 & [88] \\
\hline $\begin{array}{l}\text { Salmo trutta } \\
\text { (brown trout) }\end{array}$ & $\begin{array}{l}\text { earlier version of GGAR00000000.1 (NCBI GenBank; made } \\
\text { accessible upon request) }\end{array}$ & 62.0 & 46,308 & [88] \\
\hline \multicolumn{5}{|l|}{ Genome } \\
\hline Species & Source/Accession no. & Size $[\mathrm{Mbp}]$ & Gene count & Reference \\
\hline Salmo salar (Atlantic salmon) & GCF_000233375.1 (NCBI RefSeq assembly) & 77.5 & 48,589 & [68] \\
\hline
\end{tabular}

then performed in $H Y P H Y$ v2.3.13.20180521beta(MP) [48]. Orthogroup alignment FASTA file headers were shortened using custom Python scripts and FASTA and tree files were combined with custom $B A S H$ scripts. Using the above foreground definitions, RELAX [49], included in the HYPHY package, was run to infer orthogroups with significant signals of relaxed or intensified selection in the foreground branches relative to the background branches and to infer the selection strength parameter $k$ (as defined in [49]). The software aBSREL (adaptive Branch-Site Random-Effects Likelihood inference) [50], also contained in the HYPHY package, was then used to obtain orthogroups with a proportion of sites under significant diversifying or positive selection in the foreground branches relative to the background branches. Relevant information from both the RELAX and aBSREL output was extracted with custom $B A S H$ scripts. Multiple testing was accounted for with false-discovery rate (FDR) correction [164] or Bonferroni correction $[165,166]$ in $R$. We report on the FDR level of significance throughout the manuscript but also report the orthogroups under selection after Bonferroni correction in Additional file 1. Significant differences in the number of orthogroups under relaxed or intensified selection were quantified using Fisher's Exact Tests in $R$. A two-sided one-sample Wilcoxon signedrank test in $R$ was used to infer whether the overall selection parameter $(k)$ distribution differed significantly from the null expectation of 1 . Selection parameter distributions were visualised using the $R$ package ggplot2 [167]. Hypergeometric expectations of orthogroups under multiple types of selection were calculated in $R$.

\section{Gene ontology analyses}

GO terms were inferred using a complementary set of methods in Blast2GO v5.2.5 [156]. We used BLASTn (megablast) [168, 169] using QBlast (NCBI Blast Server) and standard web blast against non-redundant nucleotide sequences and in a second step against protein sequences. A BLAST expectation value ( $e$-value) threshold of $1.0 \mathrm{E}-3$ was used. Only the top blast hit was retained per orthogroup BLAST search. BLAST descriptors were extracted for later annotations. BLASTn was run with a word size of 28, a low complexity filter, a high-scoring segment pair (HSP) length cutoff of 33, and an HSP-hit coverage of 0 . GO mapping was performed with the Goa version 2018.12 database [170]. Orthogroups were remapped with InterProScan [171-173] using EMBL-EBI InterPro to improve mapping success. Annotations were then created using an annotation cut-off of 55, GO weight of 5 , an $e$-value filter of 1.0E-6, an HSP-hit coverage cut-off of 0 , and a hit filter of 500. Further annotations were obtained using merging of InterProScan GOs with existing annotations. Annotations were then augmented by ANNEX [174]. Remaining 
unannotated sequences were annotated using blast descriptions, applying a minimum similarity of 85 and validation of annotations. Gene functions were inferred based on Blas$t 2 G O$ and Uniprot/Swiss-Prot gene information for fish, mice, and humans (last accessed on 27/01/2019).

GO term enrichment analyses were performed using Fisher's Exact Tests in Blast2GO on the whole dataset and all sets of orthogroups under selection (from RELAX and $a B S R E L$ with Bonferroni multiple correction, FDR correction, or no correction) with FDR multiple correction (FDR threshold of 0.10). GO terms were extracted from the Blast $2 G O$ output using custom $R$ scripts. Overlap of sets of orthogroups under selection was determined with custom $R$ scripts. Additionally, GO term enrichment was tested using custom $R$ scripts to see trends in over- and underrepresentation (Blast2GO only outputs significantly over- and underrepresented GO terms after FDR correction). Violin plots of selection parameter $k$ distributions, compared across GO terms, were plotted with the ggplot $R$ package. The significance of deviations from the null expectation of $k=1$ was tested for each GO term using two-sided onesample Wilcoxon signed-rank tests in $R$.

To summarise and visualise the obtained GO terms in the different sets of orthogroups under selection, we used the REVIGO online analysis tool with an allowed similarity score of GO terms of 0.9 , the zebrafish reference GO term database available in REVIGO, and SimRel as semantic similarity measure (last access: 01.10.2019) [70], which makes use of a semantic clustering algorithm and the $p$-values from the GO term enrichment analysis in $R$ above. In this context, semantic similarity refers to the degree of relatedness between two entities/GO terms by the similarity in meaning of their annotations [175]. REVIGO clusters GO terms of sets of genes based on this algorithm in a twodimensional semantic similarity space. Additionally, REVIGO ranks the GO terms of genes according to their redundancy.

\section{Gene set enrichment analysis}

Gene set enrichment analysis (GSEA) was performed on gene symbols of zebrafish homologues of human genes derived from Blast2GO. Zebrafish homologues were obtained from http://www.informatics.jax.org/downloads/ reports/HOM_AllOrganism.rpt using a custom $R$ script. The selection parameter $(k)$ values from the above RELAX analysis were transformed into -1 to 1 scores in $R$, with negative values representing relaxed selection orthogroups and positive values representing intensified selection orthogroups. We then used WebGestalt [176-179] on the zebrafish gene symbols and associated transformed $k$ scores to derive a) the top ten enriched biological process GO terms and b) the top ten enriched KEGG pathways in both the relaxed and intensified orthogroups. We performed 10,000 permutations for each GSEA run. All other parameters were kept at default settings (last use: 02.10.2019).

\section{Supplementary information}

Supplementary information accompanies this paper at https://doi.org/10. 1186/s12864-019-6361-2

Additional file 1. Total set of orthogroups with associated GO terms and results of relaxed and intensified selection analyses.

Additional file 2. Orthogroups under diversifying selection $(F D R<0.10$ ) in both Coregonus and Salvelinus.

\section{Abbreviations}

aBSREL: Adaptive branch-site random effects likelihood; AGE: Advanced glycation end products; BASH: Bourne-again shell; BLAST: Basic local alignment search tool; BLASTn: Nucleotide basic local alignment Search Tool; bp: Base pair; dN/dS: Ratio of the number of nonsynonymous substitutions per nonsynonymous site to the number of synonymous substitutions per synonymous site; DNA: Deoxyribonucleic acid; e-value: Expectation value in BLAST search; FASTA: Text-based format for representing nucleotide and amino acid sequences; FDR: False discovery rate; FOX: Forkhead box; GO: Gene ontology; GSEA: Gene set enrichment analysis; HSP: High-scoring segment pair; KEGG: Kyoto Encyclopedia of Genes and Genomes;

Mbp: Megabase pair; Mya: Million years ago; NCBI: National Center for Biotechnology Information; ORF: Open reading frame; RAGE: Receptor for advanced glycation end products; RELAX: Hypothesis testing framework for detection of relaxed selection; RNAseq: Ribonucleic acid sequencing; SwissProt: An annotated protein sequence database; UniProt: Universal protein resource; WGD: Whole-genome duplication; $\omega$ : Synonymous to dN/dS

\section{Acknowledgements}

We thank lab members A. Yurchenko, M. Carruthers, H. Recknagel, and A. Jacobs for discussions and support during the project and M. Carruthers for contributing unpublished data.

\section{Authors' contributions}

KS and KRE designed the study with input from CEA. KS analysed the data and created the figures and tables. KS wrote the manuscript, with contributions from KRE and comments from CEA. All authors read and approved the final manuscript.

\section{Funding}

We thank the ERASMUS+ Internship programme and the Fisheries Society of the British Isles PhD Studentship programme for funding awarded to KS and Wellcome Trust-Glasgow Polyomics ISSF Catalyst funding to KRE (Wellcome Trust [097821/Z/11/Z]). The funding bodies played no role in the design of the study and collection, analysis, and interpretation of data and in writing the manuscript.

\section{Availability of data and materials}

The scripts used for orthogroup inference, selection, and gene ontology analyses, the scripts for intermediate steps and running software, and the orthogroup alignments are made publically available in the University of Glasgow Enlighten repository, DOI: https://doi.org/10.5525/gla.researchdata. 913. The transcriptomes and genomes used are from the National Center for Biotechnology Information (NCBl; https://www.ncbi.nlm.nih.gov/) (GenBank accession: GATF00000000.1, Gene Expression Omnibus accession: GSE59756, RefSeq assembly accession: GCF_000233375.1), the Oregon State University's Salmon Transcriptome website (salmon.cgrb.oregonstate.edu; Oncorhynchus mykiss), the PhyloFish project website (phylofish.sigenae.org; Coregonus clupeaformis, Salvelinus fontinalis, Thymallus thymallus, last access: 05.10.2016), and in-house data (earlier versions of NCBI GenBank accessions: GGAO00000000.1, GGAP00000000.1, GGAQ00000000.1, GGAR00000000.1; available upon request). 


\section{Consent for publication}

Not applicable.

\section{Competing interests}

The authors declare that they have no competing interests.

\section{Author details}

${ }^{1}$ Institute of Biodiversity, Animal Health \& Comparative Medicine, College of Medical, Veterinary \& Life Sciences, University of Glasgow, Glasgow G12 8Q0 UK. ${ }^{2}$ Scottish Centre for Ecology and the Natural Environment, University of Glasgow, Rowardennan G63 OAW, UK.

Received: 18 June 2019 Accepted: 1 December 2019

Published online: 23 December 2019

\section{References}

1. Manceau M, Domingues VS, Linnen CR, Rosenblum EB, Hoekstra HE. Convergence in pigmentation at multiple levels: mutations, genes and function. Philos Trans R Soc B. 2010;365:2439-50.

2. Elmer KR, Meyer A. Adaptation in the age of ecological genomics: insights from parallelism and convergence. Trends Ecol Evol. 2011;26:298-306.

3. Berner $D$, Salzburger $W$. The genomics of organismal diversification illuminated by adaptive radiations. Trends Genet. 2015;31:491-9.

4. Brawand D, Wagner CE, Li Yl, Malinsky M, Keller I, Fan S, et al. The genomic substrate for adaptive radiation in African cichlid fish. Nature. 2014;513:375-81.

5. Foote AD, Liu Y, Thomas GW, Vinař T, Alföldi J, Deng J, et al. Convergent evolution of the genomes of marine mammals. Nat Genet. 2015;47:272-5.

6. Torres-Dowdall J, Henning F, Elmer KR, Meyer A. Ecological and lineage specific factors drive the molecular evolution of rhodopsin in cichlid fishes. Mol Biol Evol. 2015;32:2876-82.

7. Castoe TA, de Koning AJ, Kim HM, Gu W, Noonan BP, Naylor G, et al. Evidence for an ancient adaptive episode of convergent molecular evolution. Proc Natl Acad Sci. 2009;106:8986-91.

8. Dobler S, Dalla S, Wagschal V, Agrawal AA. Community-wide convergent evolution in insect adaptation to toxic cardenolides by substitutions in the Na, K-ATPase. Proc Natl Acad Sci. 2012;109:13040-5.

9. Bailey SF, Rodrigue N, Kassen R. The effect of selection environment on the probability of parallel evolution. Mol Biol Evol. 2015;32:1436-48.

10. Farhat MR, Shapiro BJ, Kieser KJ, Sultana R, Jacobson KR, Victor TC, et al. Genomic analysis identifies targets of convergent positive selection in drugresistant Mycobacterium tuberculosis. Nat Genet. 2013;45:1183-9.

11. Laayouni $H$, Oosting M, Luisi P, loana M, Alonso S, Ricaño-Ponce I, et al. Convergent evolution in European and Rroma populations reveals pressure exerted by plague on Toll-like receptors. Proc Natl Acad Sci. 2014;111:2668-73.

12. Chikina M, Robinson JD, Clark NL. Hundreds of genes experienced convergent shifts in selective pressure in marine mammals. Mol Biol Evol. 2016;33:2182-92.

13. Castiglione GM, Schott RK, Hauser FE, Chang BS. Convergent selection pressures drive the evolution of rhodopsin kinetics at high altitudes via nonparallel mechanisms. Evolution. 2018;72:170-86.

14. Hatfield T, Schluter D. Ecological speciation in sticklebacks: environmentdependent hybrid fitness. Evolution. 1999;53:866-73.

15. Berner D, Grandchamp AC, Hendry AP. Variable progress toward ecological speciation in parapatry: stickleback across eight lake-stream transitions. Evolution. 2009;63:1740-53.

16. Schluter D, Conte GL. Genetics and ecological speciation. Proc Natl Acad Sci. 2009;106:9955-62.

17. Houle D, Hughes KA, Hoffmaster DK, Ihara J, Assimacopoulos S, Charlesworth B. The effects of spontaneous mutation on quantitative traits. I Variances and covariances of life history traits. Genetics. 1994;138:773-85.

18. Yoder JB, Clancey E, Des Roches S, Eastman JM, Gentry L, Godsoe W, et al. Ecological opportunity and the origin of adaptive radiations. J Evol Biol. 2010;23:1581-96.

19. Hunt BG, Ometto L, Wurm Y, Shoemaker D, Soojin VY, Keller L, Goodisman MA. Relaxed selection is a precursor to the evolution of phenotypic plasticity. Proc Natl Acad Sci. 2011;108:15936-41.

20. Velotta JP, McCormick SD, O'Neill RJ, Schultz ET. Relaxed selection causes microevolution of seawater osmoregulation and gene expression in landlocked alewives. Oecologia. 2014;175:1081-92.
21. Elmer KR, Fan S, Gunter HM, Jones JC, Boekhoff S, Kuraku S, Meyer A. Rapid evolution and selection inferred from the transcriptomes of sympatric crater lake cichlid fishes. Mol Ecol. 2010;19:197-211.

22. Fan S, Elmer KR, Meyer A. Positive Darwinian selection drives the evolution of the morphology-related gene, EPCAM, in particularly species-rich lineages of African cichlid fishes. J Mol Evol. 2011;73:1-9.

23. Wagner CE, Harmon LJ, Seehausen O. Ecological opportunity and sexual selection together predict adaptive radiation. Nature. 2012;487:366-9.

24. Recknagel H, Elmer KR, Meyer A. Crater lake habitat predicts morphological diversity in adaptive radiations of cichlid fishes. Evolution. 2014;68:2145-55.

25. Koblmüller S, Odhiambo EA, Sinyinza D, Sturmbauer C, Sefc KM. Big fish, little divergence: phylogeography of Lake Tanganyika's giant cichlid, Boulengerochromis microlepis. Hydrobiologia. 2015;748:29-38.

26. Arbour JH, López-Fernández H. Continental cichlid radiations: functional diversity reveals the role of changing ecological opportunity in the Neotropics. Proc R Soc B. 2016;283:20160556.

27. Stroud JT, Losos JB. Ecological opportunity and adaptive radiation. Annu Rev Ecol Evol Syst. 2016;47:507-32.

28. Matthews WJ. Patterns in freshwater fish ecology. New York: The University of Chicago Press; 1998.

29. Stearns SC, Hendry AP. The salmonid contribution to key issues in evolution. Evolution illuminated: Salmon and their relatives. 2004;3-19.

30. Griffiths D. The direct contribution of fish to lake phosphorus cycles. Ecol Freshw Fish. 2006;15:86-95.

31. Bernatchez L, Wilson CC. Comparative phylogeography of Nearctic and Palearctic fishes. Mol Ecol. 1998;7:431-52.

32. Elmer KR, Reggio C, Wirth T, Verheyen E, Salzburger W, Meyer A. Pleistocene desiccation in East Africa bottlenecked but did not extirpate the adaptive radiation of Lake Victoria haplochromine cichlid fishes. Proc Natl Acad Sci U S A. 2009;106:13404-9.

33. López-Fernández H, Arbour JH, Winemiller K, Honeycutt RL. Testing for ancient adaptive radiations in Neotropical cichlid fishes. Evolution. 2013;67: 1321-37.

34. Matschiner M, Musilová Z, Barth JM, Starostová Z, Salzburger W, Steel M, Bouckaert R. Bayesian phylogenetic estimation of clade ages supports transAtlantic dispersal of cichlid fishes. Syst Biol. 2017;66:3-22.

35. Primmer CR. Genetics of local adaptation in salmonid fishes. Heredity. 2011; 106:401.

36. Dodson JJ, Aubin-Horth N, Thériault V, Páez DJ. The evolutionary ecology of alternative migratory tactics in salmonid fishes. Biol Rev. 2013;88:602-25.

37. Filteau M, Pavey SA, St-Cyr J, Bernatchez L. Gene coexpression networks reveal key drivers of phenotypic divergence in lake whitefish. Mol Biol Evol. 2013;30:1384-96.

38. Chavarie L, Muir AM, Zimmerman MS, Baillie SM, Hansen MJ, Nate NA, et al. Challenge to the model of lake charr evolution: shallow-and deep-water morphs exist within a small postglacial lake. Biol J Linn Soc. 2016;120:578603.

39. Elmer KR. Genomic tools for new insights to variation, adaptation, and evolution in the salmonid fishes: a perspective for charr. Hydrobiologia. 2016;783:191-208

40. Laporte M, Dalziel AC, Martin N, Bernatchez L. Adaptation and acclimation of traits associated with swimming capacity in Lake whitefish (Coregonus clupeaformis) ecotypes. BMC Evol Biol. 2016;16:160.

41. Macqueen DJ, Primmer CR, Houston RD, et al. Functional annotation of all salmonid genomes (FAASG): an international initiative supporting future salmonid research, conservation and aquaculture. BMC Genomics. 2017;18:484

42. Moore JS, Harris LN, Le Luyer J, Sutherland BJ, Rougemont Q, Tallman RF, et al. Genomics and telemetry suggest a role for migration harshness in determining overwintering habitat choice, but not gene flow, in anadromous Arctic char. Mol Ecol. 2017;26:6784-800.

43. Jonsson B, Jonsson N. Polymorphism and speciation in Arctic charr. J Fish Biol. 2001;58:605-38

44. Kottelat M, Freyhof J. Handbook of European freshwater fishes. Cornol and Freyhof, Berlin: Publications Kottelat; 2007.

45. Vonlanthen $P$, Roy $D$, Hudson AG, Largiadèr CR, Bittner D, Seehausen O. Divergence along a steep ecological gradient in lake whitefish (Coregonus sp.). J Evol Biol. 2009;22:498-514.

46. Bernatchez L, Renaut S, Whiteley AR, Derome N, Jeukens J, Landry L, Lu G, Nolte AW, Østbye K, Rogers SM, St-Cyr J. On the origin of species: insights from the ecological genomics of the lake whitefish. Philos Trans R Soc Lond B. 2010;365:1783-800. 
47. Muir AM, Hansen MJ, Bronte CR, Krueger CC. If Arctic charr Salvelinus alpinus is 'the most diverse vertebrate', what is the lake charr Salvelinus namaycush? Fish Fish. 2016;17:1194-207.

48. Pond SLK, Muse SV. HyPhy: hypothesis testing using phylogenies. In: Statistical Methods in Molecular Evolution. New York, NY: Springer; 2005. p. 125-81.

49. Wertheim JO, Murrell B, Smith MD, Kosakovsky Pond SL, Scheffler K. RELAX: detecting relaxed selection in a phylogenetic framework. Mol Biol Evol. 2014;32:820-32

50. Smith MD, Wertheim JO, Weaver S, Murrell B, Scheffler K, Kosakovsky Pond SL. Less is more: an adaptive branch-site random effects model for efficient detection of episodic diversifying selection. Mol Biol Evol. 2015;32:1342-53.

51. Lamichhaney S, Fan G, Widemo F, Gunnarsson U, Thalmann DS, Hoeppner $M P$, et al. Structural genomic changes underlie alternative reproductive strategies in the ruff (Philomachus pugnax). Nat Genet. 2016:48:84.

52. Morandin C, Tin MM, Abril S, Gómez C, Pontieri L, Schiøtt M, et al. Comparative transcriptomics reveals the conserved building blocks involved in parallel evolution of diverse phenotypic traits in ants. Genome Biol. 2016; 17:43.

53. Sharma V, Hecker N, Roscito JG, Foerster L, Langer BE, Hiller M. A genomics approach reveals insights into the importance of gene losses for mammalian adaptations. Nat Commun. 2018;9:1215.

54. Finseth FR, Bondra E, Harrison RG. Selective constraint dominates the evolution of genes expressed in a novel reproductive gland. Mol Biol Evol. 2014;31:3266-81

55. Afanasyeva A, Bockwoldt M, Cooney CR, Heiland I, Gossmann TI. Human long intrinsically disordered protein regions are frequent targets of positive selection. Genome Res. 2018;28:975-82.

56. Yang Z, Nielsen R. Codon-substitution models for detecting molecular adaptation at individual sites along specific lineages. Mol Biol Evol. 2002;19: 908-17

57. Bielawski JP, Yang Z. Positive and negative selection in the DAZ gene family. Mol Biol Evol. 2001;18:523-9.

58. Murrell B, Weaver S, Smith MD, Wertheim JO, Murrell S, Aylward A, et al. Gene-wide identification of episodic selection. Mol Biol Evol. 2015;32:136571.

59. Orr HA, Masly JP, Presgraves DC. Speciation genes. Curr Opin Genet Dev. 2004;14:675-9.

60. Benderoth $M$, Textor S, Windsor AJ, Mitchell-Olds T, Gershenzon J, Kroymann J. Positive selection driving diversification in plant secondary metabolism. Proc Natl Acad Sci. 2006;103:9118-23.

61. Hudson AG, Lundsgaard-Hansen B, Lucek K, Vonlanthen P, Seehausen O. Managing cryptic biodiversity: fine-scale intralacustrine speciation along a benthic gradient in Alpine whitefish (Coregonus spp.). Evol Appl. 2017;10: 251-66.

62. Adams CE, Fraser D, Huntingford FA, Greer RB, Askew CM, Walker AF. Trophic polymorphism amongst Arctic charr from loch Rannoch, Scotland. J Fish Biol. 1998;52:1259-71.

63. Chavarie L, Howland K, Harris L, Tonn W. Polymorphism in lake trout in Great Bear Lake: intra-lake morphological diversification at two spatial scales. Biol J Linn Soc. 2015;114:109-25.

64. Klemetsen A, Amundsen PA, Dempson JB, Jonsson B, Jonsson N, O'Connell MF, Mortensen E. Atlantic salmon Salmo salar L., brown trout Salmo trutta L. and Arctic charr Salvelinus alpinus (L.): a review of aspects of their life histories. Ecol Freshw Fish. 2003;12:1-59.

65. McPhee MV, Utter F, Stanford JA, Kuzishchin KV, Savvaitova KA, Pavlov DS, Allendorf FW. Population structure and partial anadromy in Oncorhynchus mykiss from Kamchatka: relevance for conservation strategies around the Pacific rim. Ecol Freshw Fish. 2007;16:539-47.

66. Hodgins KA, Bock DG, Hahn MA, Heredia SM, Turner KG, Rieseberg LH. Comparative genomics in the Asteraceae reveals little evidence for parallel evolutionary change in invasive taxa. Mol Ecol. 2015;24:2226-40.

67. Macqueen DJ, Johnston IA. A well-constrained estimate for the timing of the salmonid whole genome duplication reveals major decoupling from species diversification. Proc R Soc B. 2014;281:20132881.

68. Lien S, Koop BF, Sandve SR, Miller JR, Kent MP, Nome T, et al. The Atlantic salmon genome provides insights into rediploidization. Nature. 2016;533:200-5.

69. Robertson FM, Gundappa MK, Grammes F, Hvidsten TR, Redmond AK, Lien S, Martin SAM, Holland PWH, Sandve SR, Macqueen DJ. Lineage-specific rediploidization is a mechanism to explain time-lags between genome duplication and evolutionary diversification. Genome Biol. 2017;18:111.
70. Supek F, Bošnjak M, Škunca N, Šmuc T. REVIGO summarizes and visualizes long lists of gene ontology terms. PLoS One. 2011;6:e21800.

71. Solomon KS, Kudoh T, Dawid IB, Fritz A. Zebrafish foxi1 mediates otic placode formation and jaw development. Development. 2003;130:929-40.

72. Calderoni L, Rota-Stabelli O, Frigato E, Panziera A, Kirchner S, Foulkes NS, et al. Relaxed selective constraints drove functional modifications in peripheral photoreception of the cavefish P. andruzzii and provide insight into the time of cave colonization. Heredity. 2016;117:383.

73. Škaloud P, Škaloudová M, Doskočilová P, Kim Jl, Shin W, Dvořák P. Speciation in protists: spatial and ecological divergence processes cause rapid species diversification in a freshwater chrysophyte. Mol Ecol. 2019; 28(5):1084-95. https://doi.org/10.1111/mec.15011.

74. Doenz CJ, Bittner D, Vonlanthen P, Wagner CE, Seehausen O. Rapid buildup of sympatric species diversity in Alpine whitefish. Ecol Evol. 2018;8:9398-412.

75. Siwertsson A, Knudsen R, Kahilainen K, Præbel K, Primicerio R, Amundsen PA. Sympatric diversification as influenced by ecological opportunity and historical contingency in a young species lineage of whitefish. Evol Ecol Res. 2010;12:929-47.

76. Wellborn GA, Langerhans RB. Ecological opportunity and the adaptive diversification of lineages. Ecol Evol. 2015;5:176-95.

77. Schluter D. Speciation, ecological opportunity, and latitude: (American Society of Naturalists Address). Am Nat. 2016;187:1-18.

78. Recknagel H, Hooker OE, Adams CE, Elmer KR. Ecosystem size predicts ecomorphological variability in a postglacial diversification. Ecol Evol. 2017;7: 5560-70.

79. Miller SE, Roesti M, Schluter D. A single interacting species leads to widespread parallel evolution of the stickleback genome. Curr Biol. 2019;29: 530-7 e6.

80. Shakhnovich BE, Koonin EV. Origins and impact of constraints in evolution of gene families. Genome Res. 2006;16:1529-36.

81. Roux J, Liu J, Robinson-Rechavi M. Selective constraints on coding sequences of nervous system genes are a major determinant of duplicate gene retention in vertebrates. Mol Biol Evol. 2017;34:2773-91.

82. Mitterboeck TF, Liu S, Adamowicz SJ, Fu J, Zhang R, Song W, et al. Positive and relaxed selection associated with flight evolution and loss in insect transcriptomes. GigaScience. 2017:6:1-14.

83. Roux J, Privman E, Moretti S, Daub JT, Robinson-Rechavi M, Keller L. Patterns of positive selection in seven ant genomes. Mol Biol Evol. 2014;31:1661-85.

84. Du M, Chen SL, Liu YH, Liu Y, Yang JF. MHC polymorphism and disease resistance to Vibrio anguillarum in 8 families of half-smooth tongue sole (Cynoglossus semilaevis). BMC Genet. 2011;12:78.

85. Gagnaire PA, Normandeau E, Côté C, Hansen MM, Bernatchez L. The genetic consequences of spatially varying selection in the panmictic American eel (Anguilla rostrata). Genetics. 2012;190:725-36.

86. Yan J, Zhang Y, Cheng S, Kang B, Peng J, Zhang X, et al. Common genetic heterogeneity of human interleukin-37 leads to functional variance. Cell Mol Immunol. 2017;14:783.

87. Bernatchez S, Laporte M, Perrier C, Sirois P, Bernatchez L. Investigating genomic and phenotypic parallelism between piscivorous and planktivorous lake trout (Salvelinus namaycush) ecotypes by means of RAD seq and morphometrics analyses. Mol Ecol. 2016;25:4773-92.

88. Carruthers M, Yurchenko A, Augley JJ, Adams CE, Herzyk P, Elmer KR. De novo transcriptome assembly, annotation and comparison of four ecological and evolutionary model salmonid fish species. BMC Genomics. 2018;19:32.

89. Varadharajan S, Sandve SR, Gillard GB, Tørresen OK, Mulugeta TD, Hvidsten TR, et al. The Grayling genome reveals selection on gene expression regulation after whole-genome duplication. Gen Biol Evol. 2018;10:2785-800.

90. Jacobs A, Carruthers M, Yurchenko A, Gordeeva N, Alekseyev S, Hooker O, et al. Convergence in form and function overcomes non-parallel evolutionary histories in a Holarctic fish. bioRxiv. 2019;1:265272v2.

91. Jacobs A, Carruthers M, Eckmann R, Yohannes E, Adams CE, BehrmannGodel J, Elmer KR. Rapid niche expansion by selection on functional genomic variation after ecosystem recovery. Nature Ecol Evol. 2019;3:77.

92. Perreault-Payette A, Muir AM, Goetz F, Perrier C, Normandeau E, Sirois P, Bernatchez $\mathrm{L}$. Investigating the extent of parallelism in morphological and genomic divergence among lake trout ecotypes in Lake Superior. Mol Ecol. 2017:26:1477-97.

93. Steinhäuser SS. Characterization of natterin-like genes in Arctic charr (Salvelinus alpinus). (doctoral dissertation). Reykjavíc, Iceland: University of Iceland; 2013. 
94. Gudbrandsson J, Ahi EP, Franzdottir SR, Kapralova KH, Kristjánsson BK, Steinhäuser SS, et al. The developmental transcriptome of contrasting Arctic charr (Salvelinus alpinus) morphs. F1000Research. 2015;4:136.

95. Poulin R. Greater diversification of freshwater than marine parasites of fish. Int J Parasitol. 2016;46:275-9.

96. Xing $Y$, Lee $C$. Alternative splicing and RNA selection pressure-evolutionary consequences for eukaryotic genomes. Nat Rev Genet. 2006;7:499.

97. Rottner K, Stradal TE. Actin dynamics and turnover in cell motility. Curr Opin Cell Biol. 2011;23:569-78.

98. Tan K, An L, Wang SM, Wang XD, Zhang ZN, Miao K, et al. Actin disorganization plays a vital role in impaired embryonic development of in vitro-produced mouse preimplantation embryos. PLoS One. 2015;10: e0130382.

99. Reimand J, Wagih O, Bader GD. Evolutionary constraint and disease associations of post-translational modification sites in human genomes. PLoS Genet. 2015;11:e1004919.

100. Ahi EP, Kapralova KH, Pálsson A, Maier VH, Gudbrandsson J, Snorrason SS, et al. Transcriptional dynamics of a conserved gene expression network associated with craniofacial divergence in Arctic charr. EvoDevo. 2014;5:40.

101. Ahi EP. Studies of craniofacial gene expression during embryonic development in divergent Arctic charr (Salvelinus alpinus) morphs. (doctoral dissertation). Reykjavíc, Iceland: University of Iceland; 2016.

102. Beck SV, Räsänen K, Ahi EP, Kristjánsson BK, Skúlason S, Jónsson ZO, Leblanc CA. Gene expression in the phenotypically plastic Arctic charr (Salvelinus alpinus): a focus on growth and ossification at early stages of development. Evol Dev. 2019;21:16

103. Rougeux C, Gagnaire PA, Praebel K, Seehausen O, Bernatchez L. Polygenic selection drives the evolution of convergent transcriptomic landscapes across continents within a Nearctic sister species complex. Mol Ecol. 2019; 28:4388.

104. Striberny A, Jørgensen EH, Klopp C, Magnanou E. Arctic charr brain transcriptome strongly affected by summer seasonal growth but only subtly by feed deprivation. BMC Genomics. 2019;20:529.

105. Bruneaux M, Johnston SE, Herczeg G, Merilä J, Primmer CR, Vasemägi A. Molecular evolutionary and population genomic analysis of the nine-spined stickleback using a modified restriction-site-associated DNA tag approach. Mol Ecol. 2013;22:565-82.

106. Chen X, Wang J, Yue W, Lei S, Dobjay S, Li Z, Wang C. Integrated transcriptome provides resources and insights into the adaptive evolution of colonized brown trout (Salmo trutta fario) in the Tibetan plateau. J World Aquacult Soc. 2019. https://doi.org/10.1111/jwas.12621.

107. Dennenmoser S, Vamosi SM, Nolte AW, Rogers SM. Adaptive genomic divergence under high gene flow between freshwater and brackish-water ecotypes of prickly sculpin (Cottus asper) revealed by Pool-Seq. Mol Ecol. 2017;26:25-42

108. Velotta JP, Wegrzyn JL, Ginzburg S, Kang L, Czesny S, O'Neill RJ, et al. Transcriptomic imprints of adaptation to fresh water: parallel evolution of osmoregulatory gene expression in the alewife. Mol Ecol. 2017;26:831-48.

109. Suzuki Y, Nei M. False-positive selection identified by ML-based methods: examples from the Sig1 gene of the diatom Thalassiosira weissflogii and the tax gene of a human T-cell lymphotropic virus. Mol Biol Evol. 2004;21:914-21.

110. Nozawa M, Suzuki Y, Nei M. Reliabilities of identifying positive selection by the branch-site and the site-prediction methods. Proc Natl Acad Sci. 2009; 106:6700-5.

111. Jordan G, Goldman N. The effects of alignment error and alignment filtering on the sitewise detection of positive selection. Mol Biol Evol. 2011;29:1125-39.

112. Markova-Raina $P$, Petrov D. High sensitivity to aligner and high rate of false positives in the estimates of positive selection in the 12 Drosophila genomes. Genome Res. 2011;21:863-74.

113. Zhang J. Frequent false detection of positive selection by the likelihood method with branch-site models. Mol Biol Evol. 2004;21:1332-9.

114. Zhang J, Nielsen R, Yang Z. Evaluation of an improved branch-site likelihood method for detecting positive selection at the molecular level. Mol Biol Evol. 2005;22:2472-9.

115. Yang Z. PAML 4: phylogenetic analysis by maximum likelihood. Mol Biol Evol. 2007;24:1586-91.

116. Shinde SS, Teekas L, Sharma S, Vijay N. Signatures of relaxed selection in the CYP8B1 gene of birds and mammals. J Mol Evol. 2019;87:209-20.

117. Takahashi D, Hase K, Kimura S, Nakatsu F, Ohmae M, Mandai Y, et al. The epithelia-specific membrane trafficking factor AP-1B controls gut immune homeostasis in mice. Gastroenterology. 2011;141:621-32.
118. Zizioli D, Forlanelli E, Guarienti M, Nicoli S, Fanzani A, Bresciani R, et al. Characterization of the AP-1 $\mu 1 \mathrm{~A}$ and $\mu 1 \mathrm{~B}$ adaptins in zebrafish (Danio rerio). Dev Dyn. 2010;239:2404-12.

119. Siwertsson A, Refsnes B, Frainer A, Amundsen PA, Knudsen R. Divergence and parallelism of parasite infections in Arctic charr morphs from deep and shallow lake habitats. Hydrobiologia. 2016;783:131-43.

120. Brunner FS, Anaya-Rojas JM, Matthews B, Eizaguirre C. Experimental evidence that parasites drive eco-evolutionary feedbacks. Proc Natl Acad Sci. 2017;114:3678-83.

121. Lu G, Bernatchez L. Correlated trophic specialization and genetic divergence in sympatric lake whitefish ecotypes (Coregonus clupeaformis): support for the ecological speciation hypothesis. Evolution. 1999;53:1491-505.

122. Hooker OE, Barry J, Van Leeuwen TE, Lyle A, Newton J, Cunningham P, Adams CE. Morphological, ecological and behavioural differentiation of sympatric profundal and pelagic Arctic charr (Salvelinus alpinus) in loch Dughaill Scotland. Hydrobiologia. 2016;783:209-21.

123. Braissant $\mathrm{O}$, Bachmann $\mathrm{C}$, Henry $H$. Expression and function of AGAT, GAMT and CT1 in the mammalian brain. In: Creatine and creatine kinase in health and disease. Dordrecht: Springer; 2007. p. 67-81.

124. Borchel A, Verleih M, Rebl A, Kühn C, Goldammer T. Creatine metabolism differs between mammals and rainbow trout (Oncorhynchus mykiss). SpringerPlus. 2014;3:510.

125. Borchel A, Verleih M, Kühn C, Rebl A, Goldammer T. Evolutionary expression differences of creatine synthesis-related genes: implications for skeletal muscle metabolism in fish. Sci Rep. 2019;9:5429.

126. Feng J, Chi P, Blanpied TA, Xu Y, Magarinos AM, Ferreira A, et al. Regulation of neurotransmitter release by synapsin III. J Neurosci. 2002;22:4372-80.

127. Garbarino G, Costa S, Pestarino M, Candiani S. Differential expression of synapsin genes during early zebrafish development. Neuroscience. 2014;280:351-67.

128. Kao HT, Li P, Chao HM, Janoschka S, Pham K, Feng J, et al. Early involvement of synapsin III in neural progenitor cell development in the adult hippocampus. J Comp Neurol. 2008:507:1860-70.

129. Saftig P, Hunziker E, Wehmeyer O, Jones S, Boyde A, Rommerskirch W, et al. Impaired osteoclastic bone resorption leads to osteopetrosis in cathepsin-Kdeficient mice. Proc Natl Acad Sci. 1998;95:13453-8.

130. To T. Witten PE, Huysseune A, Winkler C. An adult osteopetrosis model in medaka reveals the importance of osteoclast function for bone remodeling in teleost fish. Comp Biochem Physiol C Toxicol Pharmacol. 2015;178:68-75.

131. Kapralova KH, Gudbrandsson J, Reynisdottir S, Santos CB, Baltanás VC, Maier $\mathrm{VH}$, et al. Differentiation at the MHClla and Cath2 loci in sympatric Salvelinus alpinus resource morphs in Lake Thingvallavatn. PLoS One. 2013;8:e69402.

132. Conejeros P, Phan A, Power M, O'Connell M, Alekseyev S, Salinas I, Dixon B. Differentiation of sympatric Arctic char morphotypes using major histocompatibility class II genes. Trans Am Fish Soc. 2014;143:586-94.

133. Johnston $S E$, Orell $P$, Pritchard VL, Kent MP, Lien S, Niemelä E, et al. Genome-wide SNP analysis reveals a genetic basis for sea-age variation in a wild population of Atlantic salmon (Salmo salar). Mol Ecol. 2014;23:3452-68.

134. Gillard G, Harvey TN, Gjuvsland A, Jin Y, Thomassen M, Lien S, et al. Lifestage-associated remodelling of lipid metabolism regulation in Atlantic salmon. Mol Ecol. 2018;27:1200-13.

135. Jacobs A, Womack R, Chen M, Gharbi K, Elmer KR. Significant synteny and colocalization of ecologically relevant quantitative trait loci within and across species of salmonid fishes. Genetics. 2017;207:741-54.

136. Larson WA, Dann TH, Limborg MT, McKinney GJ, Seeb JE, Seeb LW. Parallel signatures of selection at genomic islands of divergence and the $\mathrm{MHC}$ in ecotypes of sockeye salmon across Alaska. Mol Ecol. 2019. https://doi.org/ 10.1111/mec.15082.

137. Jeukens J, Renaut S, St-Cyr J, Nolte AW, Bernatchez L. The transcriptomics of sympatric dwarf and normal lake whitefish (Coregonus clupeaformis spp., Salmonidae) divergence as revealed by next-generation sequencing. Mol Ecol. 2010;19:5389-403.

138. Bernatchez L, Renaut S, Whiteley AR, Derome N, Jeukens J, Landry L, et al. On the origin of species: insights from the ecological genomics of lake whitefish. Philos Trans R Soc B Biol Sci. 2010;365:1783-800.

139. Lynch $M$, Conery JS. The evolutionary fate and consequences of duplicate genes. Science. 2000;290:1151-5.

140. Kondrashov FA, Rogozin IB, Wolf YI, Koonin EV. Selection in the evolution of gene duplications. Genome Biol. 2002;3:8.1-9.

141. Brunet FG, Crollius HR, Paris M, Aury JM, Gibert P, Jaillon O, et al. Gene loss and evolutionary rates following whole-genome duplication in teleost fishes. Mol Biol Evol. 2006;23:1808-16. 
142. Taylor JS, Van de Peer Y, Meyer A. Genome duplication, divergent resolution and speciation. Trends Genet. 2001;17:299-301.

143. Hoegg S, Brinkmann H, Taylor JS, Meyer A. Phylogenetic timing of the fishspecific genome duplication correlates with the diversification of teleost fish. J Mol Evol. 2004;59:190-203.

144. Phillips RB, Keatley KA, Morasch MR, Ventura AB, Lubieniecki KP, Koop BF, et al. Assignment of Atlantic salmon (Salmo salar) linkage groups to specific chromosomes: conservation of large syntenic blocks corresponding to whole chromosome arms in rainbow trout (Oncorhynchus mykiss). BMC Genet. 2009;10:46.

145. Crête-Lafrenière A, Weir LK, Bernatchez L. Framing the Salmonidae family phylogenetic portrait: a more complete picture from increased taxon sampling. PLoS One. 2012;7:e46662.

146. Santini F, Harmon L, Carnevale G, Alfaro ME. Did genome duplication drive the origin of teleosts? A comparative study of diversification in ray-finned fishes. BMC Evol Biol. 2009;9:194.

147. Innan H, Kondrashov F. The evolution of gene duplications: classifying and distinguishing between models. Nat Rev Genet. 2010;11:97.

148. Lecaudey LA, Schliewen UK, Osinov AG, Taylor EB, Bernatchez L, Weiss SJ. Inferring phylogenetic structure, hybridization and divergence times within Salmoninae (Teleostei: Salmonidae) using RAD-sequencing. Mol Phylogenet Evol. 2018;124:82-99.

149. Sandve SR, Rohlfs RV, Hvidsten TR. Subfunctionalization versus neofunctionalization after whole-genome duplication. Nat Genet. 2018;50: 908-9.

150. Rosado A, Raikhel NV. Application of the gene dosage balance hypothesis to auxin-related ribosomal mutants in Arabidopsis. Plant Signal Behav. 2010; 5:450-2.

151. Birchler JA, Veitia RA. Gene balance hypothesis: connecting issues of dosage sensitivity across biological disciplines. Proc Natl Acad Sci. 2012;109:14746-53.

152. Pasquier J, Cabau C, Nguyen T, Jouanno E, Severac D, Braasch I, et al. Gene evolution and gene expression after whole genome duplication in fish: the PhyloFish database. BMC Genomics. 2016;17:368.

153. Rondeau EB, Minkley DR, Leong JS, Messmer AM, Jantzen JR, von Schalburg $\mathrm{KR}$, et al. The genome and linkage map of the northern pike (Esox lucius): conserved synteny revealed between the salmonid sister group and the Neoteleostei. PLoS One. 2014;9:e102089.

154. Fox SE, Christie MR, Marine M, Priest HD, Mockler TC, Blouin MS. Sequencing and characterization of the anadromous steelhead (Oncorhynchus mykiss) transcriptome. Mar Genomics. 2014;15:13-5.

155. Tomalty KM, Meek MH, Stephens MR, Rincón G, Fangue NA, May BP, Baerwald MR. Transcriptional response to acute thermal exposure in juvenile Chinook salmon determined by RNAseq. G3: Genes. Genomes, Genetics. 2015:5:1335-49.

156. Conesa A, Götz S, García-Gómez JM, Terol J, Talón M, Robles M. Blast2GO: a universal tool for annotation, visualization and analysis in functional genomics research. Bioinformatics. 2005;21:3674-6.

157. Emms DM, Kelly S. OrthoFinder: solving fundamental biases in whole genome comparisons dramatically improves orthogroup inference accuracy. Genome Biol. 2015;16:157.

158. Löytynoja A. Phylogeny-aware alignment with PRANK. In Multiple Sequence Alignment Methods. Totowa, NJ: Humana Press; 2014. p. 155-70.

159. Capella-Gutiérrez S, Silla-Martínez JM, Gabaldón T. trimAl: a tool for automated alignment trimming in large-scale phylogenetic analyses. Bioinformatics. 2009;25:1972-3.

160. Lefort V, Desper R, Gascuel O. FastME 2.0: a comprehensive, accurate, and fast distance-based phylogeny inference program. Mol Biol Evol. 2015;32: 2798-800.

161. R Core Team. R: a language and environment for statistical computing. Vienna, Austria: R Foundation for Statistical Computing; 2016. https://www. R-project.org/

162. Paradis E, Claude J, Strimmer K. APE: analyses of phylogenetics and evolution in R language. Bioinformatics. 2004;20:289-90.

163. Popescu AA, Huber KT, Paradis E. Ape 3.0: new tools for distance-based phylogenetics and evolutionary analysis in R. Bioinformatics. 2012;28:1536-7.

164. Benjamini $Y$, Hochberg Y. Controlling the false discovery rate: a practical and powerful approach to multiple testing. J R Stat Soc Ser B Methodol. 1995:57:289-300

165. Bonferroni C. Teoria statistica delle classi e calcolo delle probabilita. Pubblicazioni del R Istituto Superiore di Scienze Economiche e Commericiali di Firenze. 1936:8:3-62.
166. Dunn OJ. Multiple comparisons among means. J Am Stat Assoc. 1961;56: 52-64.

167. Wickham H. ggplot2. Wiley Interdisciplinary Reviews: Computational Statistics. 2011;3:180-5.

168. Altschul SF, Gish W, Miller W, Myers EW, Lipman DJ. Basic local alignment search tool. J Mol Biol. 1990;215:403-10.

169. Morgulis A, Coulouris G, Raytselis Y, Madden TL, Agarwala R, Schäffer AA. Database indexing for production MegaBLAST searches. Bioinformatics. 2008;24:1757-64.

170. Camon E, Magrane M, Barrell D, Lee V, Dimmer E, Maslen J, et al. The gene ontology annotation (Goa) database: sharing knowledge in uniprot with gene ontology. Nucleic Acids Res. 2004;32:D262-6.

171. Zdobnov EM, Apweiler R. InterProScan - an integration platform for the signature-recognition methods in InterPro. Bioinformatics. 2001;17:847-8.

172. Quevillon E, Silventoinen V, Pillai S, Harte N, Mulder N, Apweiler R, Lopez R. InterProScan: protein domains identifier. Nucleic Acids Res. 2005;33:W116-20.

173. Jones $P$, Binns D, Chang HY, Fraser M, Li W, McAnulla C, et al. InterProScan 5: genome-scale protein function classification. Bioinformatics. 2014;30: 1236-40.

174. Myhre S, Tveit H, Mollestad T, Lægreid A. Additional gene ontology structure for improved biological reasoning. Bioinformatics. 2006;22:2020-7.

175. Pesquita C, Faria D, Falcao AO, Lord P, Couto FM. Semantic similarity in biomedical ontologies. PLoS Comput Biol. 2009;5:e1000443.

176. Zhang B, Kirov SA, Snoddy JR. WebGestalt: an integrated system for exploring gene sets in various biological contexts. Nucleic Acids Res. 2005; 33(web server issue):W741-8.

177. Wang J, Duncan D, Shi Z, Zhang B. WEB-based GEne SeT AnaLysis toolkit (WebGestalt): update 2013. Nucleic Acids Res. 2013;41 (web server issue): W77-83.

178. Wang J, Vasaikar S, Shi Z, Greer M, Zhang B. WebGestalt 2017: a more comprehensive, powerful, flexible and interactive gene set enrichment analysis toolkit. Nucleic Acids Res. 2017;45:W130-7.

179. Liao Y, Wang J, Jaehnig E, Shi Z, Zhang B. WebGestalt 2019: gene set analysis toolkit with revamped UIs and APIs. Nucleic Acids Res. 2019;47: W199-205.

\section{Publisher's Note}

Springer Nature remains neutral with regard to jurisdictional claims in published maps and institutional affiliations.

\section{Ready to submit your research? Choose BMC and benefit from:}

- fast, convenient online submission

- thorough peer review by experienced researchers in your field

- rapid publication on acceptance

- support for research data, including large and complex data types

- gold Open Access which fosters wider collaboration and increased citations

- maximum visibility for your research: over $100 \mathrm{M}$ website views per year

At $\mathrm{BMC}$, research is always in progress.

Learn more biomedcentral.com/submissions 